\title{
Regularity in the local CR embedding problem
}

\author{
Xianghong Gong* and S. M. Webster
}

May 29, 2018

\begin{abstract}
We consider a formally integrable, strictly pseudoconvex CR manifold $M$ of hypersurface type, of dimension $2 n-1 \geq 7$. Local CR, i.e. holomorphic, embeddings of $M$ are known to exist from the works of Kuranishi and Akahori. We address the problem of regularity of the embedding in standard Hölder spaces $C^{a}(M), a \in \mathbf{R}$. If the structure of $M$ is of class $C^{m}, m \in \mathbf{Z}, 4 \leq m \leq \infty$, we construct a local $\mathrm{CR}$ embedding near each point of $M$. This embedding is of class $C^{a}$, for every $a, 0 \leq a<m+(1 / 2)$. Our method is based on Henkin's local homotopy formula for the embedded case, some very precise estimates for the solution operators in it, and a substantial modification of a previous Nash-Moser argument due to the second author.

Key Words. Tangential Cauchy-Riemann Equations, CR embedding, Nash-Moser methods.

MSC Classification. Primary 32V30; Secondary 35N10.
\end{abstract}

\section{Contents}

1 Initial normalization. Non-isotropic dilation.

2 Local real hypersurfaces in $\mathrm{C}^{n}$. 7

3 Alteration of the embedding. 9

4 Renormalization of the embedding.

5 Hölder estimates. 14

6 A general estimate for the new error. 16

7 Estimates for $S_{t}, P$, and $Q$. 18

*Partially supported by NSF grant DMS-0705426. 
8 Estimates for $F$. 19

9 Four-term estimates for $\bar{\partial}_{X}(Z+F)$. 21

10 The sequence of embeddings. Summary. 24

11 The main inductive hypotheses. 26

12 Convergence in $C^{a}$. 28

13 Interpolation and higher regularity. 30

14 Composition of mappings. 32

INTRODUCTION. In this paper we prove the following theorem: Let $M$ be a formally integrable, strongly pseudoconvex $\mathrm{CR}$ manifold of differentiability class $C^{m}, m \in \mathbf{Z}$, and dimension $2 n-1 \geq 7$. Then, near each point of $M$, there exists a local CR embedding $Z$ into $\mathbf{C}^{n}$. This embedding $Z$ is of Hölder class $C^{a}$, for every $a, 0 \leq a<m+1 / 2$.

We state the result in a more precise form below. Locally, we take $U \subset \mathbf{R}^{2 n-1}$ to be a neighborhood of the origin. The CR structure is given by $n-1$ complex vector fields, $X_{\alpha}, 1 \leq \alpha \leq n-1$ on $U$, which together with their complex conjugates $X_{\bar{\alpha}}$ are pointwise independent over $\mathbf{C}$. The Lie brackets $\left[X_{\alpha}, X_{\beta}\right]$ are linear combinations of the $X_{\alpha}$ 's, which is the integrability condition. The brackets $i\left[X_{\alpha}, X_{\bar{\beta}}\right]$, modulo the $X_{\alpha}, X_{\bar{\alpha}}$ 's give the Hermitian Levi form, which is assumed to be positive definite. The $\mathrm{CR}$ embedding $Z=\left(z^{1}, \ldots, z^{n}\right)$, is to be given by $n$ independent local complex functions near 0 , which satisfy the overdetermined system of first-order, linear partial differential equations

$$
X_{\bar{\alpha}} z^{j}=0, \quad 1 \leq \alpha \leq n-1,1 \leq j \leq n .
$$

Our main result is the following.

Theorem 0.1. Let the coefficients of the vector-fields $X_{\alpha}$ be of class $C^{m}, m \in \mathbf{Z}$, $4 \leq m \leq \infty$, and $2 n-1 \geq 7$. Then there exist $n$ independent solutions $z^{j}$ to the above system, which embed some neighborhood of 0 as a strictly pseudoconvex real hypersurface $M^{2 n-1}$ in $\mathbf{C}^{n}$. These functions $z^{j}$ are of Hölder class $C^{a}$, for all a, $0 \leq a<m+1 / 2$.

There are counterexamples to the existence of local CR embeddings in the case $2 n-1=3$, due to Nirenberg [20], while the case $2 n-1=5$ is still unresolved, to our knowledge. There are also counterexamples in the case of Levi nondegenerate structures of mixed signature [11]. 
The lower bound on $m$ can undoubtedly be improved. A reasonable conjecture would be that the theorem holds with $m \geq 2$. In fact, for many results in this work, we may take the $X_{\alpha}$ in the Hölder class $C^{m}, m \in \mathbf{R}, 3<m \leq \infty$. Then we can get a CR-embedding of class $C^{a}$, if $0 \leq a<m$. See proposition 12.1 below.

Perhaps more interestingly, it seems likely that one might achieve $a=m+1 / 2$ for ours, or for some other solution $Z$. This reminds us of the situation of the fundamental (1/2)-estimate for the $\bar{\partial}$-problem on strictly pseudoconvex domains (see Range [21]). Recall briefly that Kerzman [12] and Stein showed that one cannot gain more than Hölder $1 / 2$ in this problem, and Kerzman established a gain of $<1 / 2$. After much work, Henkin and Romanov [7], [9] proved the existence of solutions gaining precisely $1 / 2$ derivative, by kernel methods.

Henkin's construction [8] of solution operators for the local tangential CauchyRiemann equations on a strictly pseudoconvex real hypersurface $M$, together with the detailed estimates in [6], form key ingredients in the proof of the above theorem. Since these operators do not regain a full derivative, we must introduce a smoothing process. Thus, the other main ingredient is a Nash-Moser implicit function theorem [16], [17], [18].

The CR embedding problem now has a long history. The local result was first conjectured by Kohn [13], who also founded the analysis of CR manifolds using Hilbert space methods. Theorem 0.1 is an analogue of the Newlander-Nirenberg theorem (see [20]) for integrable almost-complex structures. That venerable result now has many different proofs. We mention only the one in [22], the method of which is particularly relevant to the argument given here. It motivated the proof in [24] of a much less precise version of theorem 0.1. The work of Ma and Michel [15] greatly reduced the derivative loss in that argument. We also mention another simpler but instructive model, the integrability problem for $\mathrm{CR}$ vector bundles. In [5] we were able to eliminate completely the previous complicated Nash-Moser techniques and give a simple and sharp argument based on the KAM method. To date, however, all known proofs of local CR embedding involve some kind of difficult Nash-Moser argument.

In case the CR manifold $M^{2 n-1}$ is also compact, and $2 n-1 \geq 5$, Boutet-deMonvel has given a linear proof (of local embedding) [2], based on Kohn's estimates [13]. By far the most important step toward theorem 0.1 was taken by Kuranishi [14] for $2 n-1 \geq 9$. This was extended to $2 n-1 \geq 7$ by Akahori [1]. Catlin has given another proof of even more general results [3] based on his method of extending the $\mathrm{CR}$ structure to an integrable almost complex structure. These results are all carried out in $C^{\infty}$, using $\bar{\partial}$-Neumann-type methods.

The scheme of proof of theorem 0.1 is similar to that developed in [24], but with some substantial and significant changes. We start with a smooth approximate CR (or holomorphic) embedding and modify it to make it more nearly holomorphic. We 
iterate this process, generating a sequence of smooth embeddings, which converge in suitable norm to a CR embedding with the stated regularity.

In section one we make an initial normalization of the complex vector fields $X_{\alpha}$ at 0 . This produces an approximate embedding onto a real hyperquadric in $\mathbf{C}^{n}$. Instead of using higher order Taylor polynomial arguments, we apply nonisotropic dilation to get approximate CR embeddings with arbitrarily small error. This obviates much of the spurious derivative loss encountered in [24].

In section 2 we recall the tangential CR operator $\bar{\partial}_{M}$ and local Henkin homotopy formula, with solution operators $P, Q$ for a normalized strictly pseudoconvex real hypersurface $M \subset \mathbf{C}^{n}$. By normalized we mean that $M$ is suitably approximated to second order at the origin by a real hyperquadric. Then in section 3 we indicate our basic procedure, $Z \mapsto Z_{*}=Z+F$, to make the embedding more nearly holomorphic. For this the error term $X_{\bar{\alpha}}(Z+F)$ is decomposed into a sum of 4 terms. This procedure destroys the normalization at 0 , so section 4 is needed to give a precise method for renormalization. This uses the first order Taylor polynomial of $F$. The inverse mapping theorem is then applied to the map $f$ gotten by projecting $Z \mapsto Z_{*}$ to the real hyperplane $\operatorname{Re} z^{n}=0$. The map $f$ reparametrizes the $\mathrm{CR}$ structure, and $Z_{*} \circ f^{-1}$ is the new approximate CR embedding.

This procedure is rather lengthy, but it is one for which we can make precise estimates. These are in terms of standard Hölder norms, which we recall in section 5. We make use of elementary properties of these norms in sections 5 and 6 to get estimates in terms of $F$. These estimates are roughly of two types. The "coarse" estimates will be used for controlling the growth of higher order derivatives during iteration. The "fine" estimates will be used to get rapid convergence in some lower order Hölder norm.

In section 7 we introduce a standard smoothing operator $S_{t}$ and give some well known estimates for it. Moreover, we make a precise estimate for the Friedrichs commutator $\left[S_{t}, \bar{\partial}_{M}\right]$. Also in section 7 we quote from [6] the needed estimates for the Henkin solution operators $P, Q$. In section 8 we give estimates for $F$, and use them to refine our previous estimates. Section 9 contains the central estimates, the 4-term estimates for $X_{\bar{\alpha}}(Z+F)$.

In sections 10, 11 we establish the main inductive hypotheses and construct the sequence of approximate $\mathrm{CR}$ embeddings. It is shown to converge in a low derivative norm to a $\mathrm{CR}$ embedding in section 12, provided $m>3$. This inequality results from the choice of certain parameters in the argument, and is probably not optimal. In section 13 we use the interpolation inequality for Hölder norms, the rapid convergence in low norm, and some delicate estimates controlling the possible growth of higher order derivatives, to gain increased regularity for the limiting real hypersurface $M_{\infty}$ and its embedding $Z_{\infty}$ into $\mathbf{C}^{n}$. In section 14 we show convergence of the infinite composition of the projected maps $f$ to a map $\tilde{f}_{\infty}$. The solutions to 
the system $(0.1)$ are then given by the components $z^{j}$ of $Z_{\infty} \circ \tilde{f}_{\infty}$. This will prove theorem 0.1 .

\section{Initial normalization. Non-isotropic dilation.}

We consider $n-1$ complex vector fields $X_{\alpha}, 1 \leq \alpha \leq n-1$, in a neighborhood of $0 \in \mathbf{R}^{2 n-1}$, of smoothness class $C^{m}, m \in \mathbf{Z}, m \geq 2$, which, together with their complex conjugates $X_{\bar{\alpha}}=\overline{X_{\alpha}}$, are pointwise linearly independent over the complex numbers. Under the integrability and non-degeneracy conditions, we normalize them at 0 to get a second order approximate $\mathrm{CR}$ embedding onto a real hyperquadric. Then we use non-isotropic dilation to make the error as small as we please in $C^{m}$ norm.

Lemma 1.1. After a polynomial change of the coordinates $\left(z^{\alpha}, x^{n}\right)$ on $\mathbf{R}^{2 n-1}$, we may achieve

$$
X_{\alpha}=\partial_{\alpha}+\tilde{A}_{\alpha}^{\bar{\beta}} \partial_{\bar{\beta}}+\tilde{B}_{\alpha} \partial_{x^{n}}, \quad \tilde{B}_{\alpha}=i \delta_{\alpha \bar{\beta}} z^{\bar{\beta}}+B_{\alpha}^{*}
$$

where $\tilde{A}_{\alpha}^{\bar{\beta}} \equiv B_{\alpha}^{*} \equiv O(2) \equiv O\left(\left|\left(z^{\prime}, x^{n}\right)\right|^{2}\right)$, and the coefficients are of class $C^{m}$.

Proof. We only sketch the proof, since the principle is well known. We first choose linear coordinates so that $X_{\alpha}(0)=\partial_{\alpha} \equiv \partial / \partial z^{\alpha}$. Then a frame change achieves the

form $(1.1)$ with $\tilde{A}_{\alpha}^{\bar{\beta}}(0)=0, \tilde{B}_{\alpha}(0)=0$. For such a frame the integrability condition is equivalent to the Lie brackets $\left[X_{\alpha}, X_{\beta}\right]=0$, or

$$
X_{\alpha} \tilde{A}_{\beta}^{\bar{\gamma}}=X_{\beta} \tilde{A}_{\alpha}^{\bar{\gamma}}, \quad X_{\alpha} \tilde{B}_{\beta}=X_{\beta} \tilde{B}_{\alpha}
$$

The Levi form is the hermitian matrix $g_{\alpha \bar{\beta}}$ defined by

$$
\begin{aligned}
{\left[X_{\alpha}, X_{\bar{\beta}}\right] } & \equiv-i g_{\alpha \bar{\beta}} \partial_{x^{n}}, \bmod \left\{X_{\gamma}, X_{\bar{\gamma}}\right\} \\
g_{\alpha \bar{\beta}}(0) & =i\left(X_{\alpha} \tilde{B}_{\bar{\beta}}-X_{\bar{\beta}} \tilde{B}_{\alpha}\right)(0)
\end{aligned}
$$

It is assumed to be positive definite. After a change of the frame $X_{\alpha}$, we may assume $g_{\alpha \bar{\beta}}(0)=2 \delta_{\alpha \bar{\beta}}(0)$. The symmetry in $\alpha, \beta$ in (1.2) allows us to remove certain terms using Taylor polynomials in $z^{\prime}=\left(z^{\alpha}\right)$ and get the normalization along the $x^{n}$-axis. We replace this normalizing transformation by its Taylor polynomial at 0 , to avoid losing derivatives. For more details see the first section of [24].

We now consider approximate CR, or holomorphic, embeddings of the form

$$
Z=\left(z^{\prime}, z^{n}\right), \quad z^{n}=x^{n}+i y^{n}, \quad y^{n}=y^{n}\left(z^{\prime}, x^{n}\right),
$$


onto a smooth strictly pseudoconvex real hypersurface $M \subset \mathbf{C}^{n}$, of the form

$$
M: r=0, r(z)=-y^{n}+\left|z^{\prime}\right|^{2}+h\left(z^{\prime}, x^{n}\right),
$$

where $h \equiv O(2)$ is a $C^{\infty}$-smooth real function defined on a neighborhood $D$ of 0 , and vanishing to order 2 at 0 ,

$$
0 \in D \subseteq \mathbf{R}^{2 n-1}=\left\{y^{n}=0\right\} \subset \mathbf{C}^{n} .
$$

The hypersurface and embedding will be referred to as normalized.

A basis of complex tangential $(1,0)$-vector fields to $M$ is given by

$$
Y_{\alpha}=\partial_{\alpha}-\left(r_{\alpha} / r_{n}\right) \partial_{n} \equiv \partial_{\alpha}-\left(r_{\alpha} / 2 r_{n}\right) \partial_{x^{n}}
$$

the latter being the representation on $\mathbf{R}^{2 n-1}$. The given vector fields $X_{\alpha}$ and their complex conjugates $X_{\bar{\alpha}}$, now considered on $D$, will be adapted to the embedding $Z$ by the condition $X_{\alpha} z^{\beta}=\delta_{\alpha}^{\beta}$, which determines them uniquely. We then write them as

$$
\begin{aligned}
X_{\alpha} & =Y_{\alpha}+A_{\alpha}^{\bar{\beta}} Y_{\bar{\beta}}+B_{\alpha} \partial_{x^{n}}, \\
X_{\bar{\alpha}} z^{\beta} & =A_{\bar{\alpha}}^{\beta}, \quad X_{\bar{\alpha}} z^{n}=\left(1+i \partial_{x^{n}} h\right) B_{\bar{\alpha}}-A_{\bar{\alpha}}\left(r_{\beta} / r_{n}\right) .
\end{aligned}
$$

It follows that the embedding is nearly holomorphic, to the extent that the coefficients $A_{\bar{\alpha}}^{\beta}=\overline{A_{\alpha}^{\bar{\beta}}}$, and $B_{\bar{\alpha}}=\overline{B_{\alpha}}$ are small.

With the normalization (1.1), we achieve the embedding (1.4) with $h=0$ in (1.5) and with vector fields of the form (1.7), (1.8), where $A_{\alpha}^{\bar{\beta}}=\tilde{A}_{\alpha}^{\bar{\beta}}$ and $B_{\alpha}=$ $B_{\alpha}^{*}+i A_{\alpha}^{\bar{\beta}}\left(r_{\bar{\beta}} / 2 r_{\bar{n}}\right)$. Thus, the embedding is onto a real hyperquadric $M$, with $X_{\bar{\alpha}} Z=$ $O(2)$.

The real hyperquadric $M$, and the real hyperplane $y^{n}=0$, are both invariant by the family of non-isotropic dilations, $f_{\rho}\left(z^{\prime}, z^{n}\right)=\left(\rho z^{\prime}, \rho^{2} z^{n}\right), f_{\rho}: D_{s}^{0} \rightarrow D_{\rho s}^{0}$, where $D_{s}^{0}=\left\{\left|z^{\prime}\right|^{4}+\left(x^{n}\right)^{2}<s^{4}\right\} \subset \mathbf{R}^{2 n-1}$. We take $s$ small, $s \rho=1$, replace $Z$ by $f_{\rho} \circ Z$, and the vector fields $X_{\alpha}$ by $X_{\alpha}^{(\rho)}=\rho^{-1}\left(f_{\rho}\right)_{*}\left(X_{\alpha}\right)$. Then

$$
\begin{aligned}
X_{\alpha}^{(\rho)} & =Y_{\alpha}+A_{\alpha}^{(\rho) \bar{\beta}} Y_{\bar{\beta}}+B_{\alpha}^{(\rho)} \partial_{x^{n}} \\
A_{\alpha}^{(\rho) \bar{\beta}} & =A_{\alpha}^{\bar{\beta}}\left(\rho^{-1} z^{\prime}, \rho^{-2} x^{n}\right), \quad B_{\alpha}^{(\rho)}=\rho B_{\alpha}\left(\rho^{-1} z^{\prime}, \rho^{-2} x^{n}\right) .
\end{aligned}
$$

(Note that a general dilation $x \mapsto \rho x=\left(\rho_{1} x_{1}, \ldots, \rho_{n} x_{n}\right)$ transforms a vector field $\Sigma \xi_{j}(x) \partial_{x_{j}}$ to $\left.\Sigma \xi_{j}\left(\rho^{-1} x\right) \rho_{j} \partial_{x_{j}}.\right)$ Taking any number $k \leq m$ of $\left(z^{\prime}, x^{n}\right)$-derivatives, we see that

$$
\partial^{k} A_{\alpha}^{(\rho) \bar{\beta}}=O\left(\rho^{-2}\right), \quad \partial^{k} B_{\alpha}^{(\rho)}=O\left(\rho^{-1}\right),
$$

hence $X_{\bar{\alpha}}^{(\rho)}\left[f_{\rho} \circ Z\right]=O\left(\rho^{-1}\right)$, in $C^{m}$-norm, as $\rho \rightarrow \infty$. Thus, by shrinking our original domain in $\mathbf{R}^{2 n-1}$, dilating it to unit size, and dropping the notation $(\rho)$ in (1.10), we achieve the following lemma. 
Lemma 1.2. Near any point, taken as the origin, there are approximate holomorphic embeddings $Z$, of the form (1.4) defined on the unit ball $B_{1}$ in $\mathbf{R}^{2 n-1}$, for the given $C R$ structure of class $C^{m}, m \geq 2$. Basis vector fields $X_{\alpha}$ for the structure satisfy $X_{\bar{\alpha}} Z=O(2)$, and may be taken in the form (1.8), with $X_{\bar{\alpha}} Z$ as small as we please in the $C^{m}\left(B_{1}\right)$-norm.

\section{Local real hypersurfaces in $\mathrm{C}^{n}$.}

In this section we consider a local, normalized, strictly pseudoconvex real hypersurface $M \subset \mathbf{C}^{n}$. First we determine the relevant geometric properties of $M$, under some further conditions. Then we recall the local tangential Cauchy-Riemann complex, and the local homotopy formula first given by Henkin.

To establish more precisely the needed properties of $M(1.5)$, we assume that the domain $D(1.6)$ is a convex neighborhood of 0 . We further define $x=\left(z^{\prime}, x^{n}\right)$, $|x|^{2}=\left(x^{n}\right)^{2}+z^{\prime} \cdot \bar{z}^{\prime}$, and

$$
\begin{aligned}
\psi(x) & =|x|^{2}+h(x)=\left(x^{n}\right)^{2}+\left|z^{\prime}\right|^{2}+h\left(z^{\prime}, x^{n}\right), \\
D_{\rho} & \equiv D_{\rho}(h)=\left\{x \in D \mid \psi(x) \leq \rho^{2}\right\} .
\end{aligned}
$$

By Taylor's formula, $|h(x)| \leq c_{2}|x|^{2}$ on $D$, where $c_{2}=\|h\|_{C^{2}(D)}$. We assume that $c_{2}<1$ so that

$$
\begin{aligned}
\left(1-c_{2}\right)|x|^{2} & \leq \psi(x) \\
B\left(\rho / \sqrt{1+c_{2}}\right) \cap D \quad & \subseteq D_{\rho} \quad \subseteq B\left(\rho / \sqrt{1-c_{2}}\right) \cap D,
\end{aligned}
$$

where $B(\rho)$ denotes the ball of radius $\rho$ centered at 0 . In particular, $D_{\rho}$ is contained in the interior of $D$, if $\rho>0$ is sufficiently small, which we now also assume.

To investigate the smoothness of $\partial D_{\rho}$, suppose $0=d \psi(x)$. Then $2|x|^{2}=$ $-x^{j} h_{j}(x)$, so that $2|x|^{2} \leq c_{2}|x|^{2}$; hence $x=0$. It follows that $\partial D_{\rho}$ is smooth for small $\rho>0$. Then $D_{\rho}$ will be strictly convex, if the Hessian $\left(\psi_{i j}\right)$ is positive definite on $D$. But this also holds, since $\psi_{i j}(x) v^{i} v^{j}=2|v|^{2}+h_{i j}(x) v^{i} v^{j} \geq\left(2-c_{2}\right)|v|^{2}$.

Finally, we estimate the distance between $\partial D_{\rho}$ and $\partial D_{\hat{\rho}}$, where $\hat{\rho}=\rho(1-\sigma)$, $0<\sigma<1$, assuming these to be compact, smooth, and strictly convex. Let $x_{0} \in$ $\partial D_{\hat{\rho}}$ and $x_{1} \in \partial D_{\rho}$ satisfy $\left|x_{0}-x_{1}\right|=\operatorname{dist}\left(\partial D_{\hat{\rho}}, \partial D_{\rho}\right)$. Then the segment $\left[x_{0}, x_{1}\right]$ is orthogonal to the two tangent planes, and $\left|x_{1}\right|>\left|x_{0}\right|$, since $D_{\hat{\rho}}$ is a convex neighborhood of 0 . By the Schwarz inequality and the fact that $|d h(x)| \leq c_{2}\left|x_{1}\right|$, for $x$ on the segment $\left[x_{0}, x_{1}\right]$, we have

$$
\begin{aligned}
\rho^{2}-\hat{\rho}^{2} & =\left\langle x_{1}-x_{0}, x_{1}\right\rangle+\left\langle x_{0}, x_{1}-x_{0}\right\rangle+h\left(x_{1}\right)-h\left(x_{0}\right) \\
& \leq\left|x_{1}-x_{0}\right|\left(\left|x_{0}\right|+\left|x_{1}\right|\right)+\left|x_{1}-x_{0}\right| c_{2}\left|x_{1}\right| \\
& \leq\left(2+c_{2}\right)\left|x_{1}-x_{0}\right| \cdot\left|x_{1}\right| .
\end{aligned}
$$


But by $(2.9), \rho^{2} \geq\left(1-c_{2}\right)\left|x_{1}\right|^{2} \geq(1 / 2)\left|x_{1}\right|^{2}$, provided $c_{2}<1 / 2$. Then

$$
\rho \sigma \leq \hat{c} \cdot \operatorname{dist}\left(\partial D_{\rho(1-\sigma)}, \partial D_{\rho}\right),
$$

where $\hat{c}=\sqrt{2}\left(2+c_{2}\right) \leq 3 \sqrt{2}$.

This proves the following.

Lemma 2.1. There is a constant $\gamma_{0}, 0<\gamma_{0}<1 / 2$, such that the following holds. Let $D$ in (1.6) be a convex neighborhood of 0 , and let the smooth real function $h$ as above satisfy

$$
h=O\left(|x|^{2}\right), \quad\|h\|_{C^{2}(D)}<\gamma_{0}
$$

Suppose that $\rho_{0}>0$ is so small that $D_{\rho_{0}}$ in (2.2) is contained in the interior of $D$. Then for each $0<\rho \leq \rho_{0}$, the domain $D_{\rho}$ is compact, smoothly bounded, strictly convex, and

$$
B(\sqrt{2 / 3} \rho) \subseteq D_{\rho} \subseteq B(\sqrt{2} \rho)
$$

For $0<\rho \leq \rho_{0}$ and $0<\sigma<1$, the inequality (2.6) holds.

We now consider the vector fields $Y_{\alpha}(1.7)$, and $X_{\sigma}(1.8)$, on $D$ and the corresponding tangential CR-complexes. For functions $f$ on $M$ (or on $D$ ), we define

$$
\bar{\partial}_{M} f=\sum Y_{\bar{\alpha}} f d z^{\bar{\alpha}}, \bar{\partial}_{X} f=\sum X_{\bar{\alpha}} f d z^{\bar{\alpha}}
$$

For a tangential $(0, \mathrm{q})$-form on $M, \phi=\sum \phi_{\bar{A}} d \bar{z}^{\bar{A}}$, in standard multi-index notation, $A=\left(\alpha_{1}, \ldots, \alpha_{n-1}\right), 1 \leq \alpha_{j} \leq n-1$, we set

$$
\bar{\partial}_{M} \phi=\sum \bar{\partial}_{M} \phi_{\bar{A}} \wedge d \bar{z}^{\bar{A}}, \quad \bar{\partial}_{X} \phi=\sum \bar{\partial}_{X} \phi_{\bar{A}} \wedge d \bar{z}^{\bar{A}}
$$

From the form of the vectors $X_{\alpha}, Y_{\alpha}$, and their respective integrability conditions, it follows that $\left[X_{\alpha}, X_{\beta}\right]=0$, and $\left[Y_{\alpha}, Y_{\beta}\right]=0$. This gives

$$
\left(\bar{\partial}_{M}\right)^{2}=0, \quad\left(\bar{\partial}_{X}\right)^{2}=0
$$

For $0<\rho \leq \rho_{0}$ as in the lemma, we denote by $M_{\rho}$ the part of $M$ lying over $D_{\rho}$. Thus,

$$
M_{\rho}=M \cap\left\{\left(z^{\prime}, z^{n}\right) \in \mathbf{C}^{n} \mid\left(x^{n}\right)^{2}+y^{n} \leq \rho^{2}\right\}
$$

It is a compact, smoothly bounded domain on the strictly pseudoconvex real hypersurface $M$. Its main property is that it is cut out by a real function of the 
holomorphic function, $z^{n}$. For such domains we have the local Henkin homotopy formula [8], [23], [6],

$$
\phi=\bar{\partial}_{M} P \phi+Q \bar{\partial}_{M} \phi
$$

for (0,q)-forms $\phi$ as above, where $P, Q$ involve certain integral operators over $M_{\rho}$ and its boundary. This formula is valid only for $0<q<n-2$. We use it in the case $q=1$. This requires $\operatorname{dim} M=2 n-1 \geq 7$, thus excluding the five dimensional case. This is the only point in our argument where this condition is required. In the 5 dimensional case there is an extra term added to the right hand side of (2.13). As of this writing, it remains unclear whether such a more general homotopy formula can be used. See [24] and Nagel-Rosay [19].

\section{Alteration of the embedding.}

Given an embedding $Z$ such that the error $\left\|\bar{\partial}_{X} Z\right\|=\sum\left\|X_{\bar{\alpha}} Z\right\|$ is "small", we wish to perturb it to one $Z_{*}\left(z^{\prime}, x^{n}\right)=Z\left(z^{\prime}, x^{n}\right)+F\left(z^{\prime}, x^{n}\right)$, so that the new error $\left\|\bar{\partial}_{X} Z_{*}\right\|$ is "smaller". These terms as well as the norms will be made precise later.

We assume the results of the previous section, and apply the homotopy formula (2.13) component-wise to $\phi=\bar{\partial}_{X} Z$, to get

$$
\begin{aligned}
\bar{\partial}_{X} Z_{*} & =\bar{\partial}_{X} Z+\bar{\partial}_{M} F+\left(\bar{\partial}_{X}-\bar{\partial}_{M}\right) F \\
& =\bar{\partial}_{M}\left(P \bar{\partial}_{X} Z+F\right)+Q\left(\bar{\partial}_{M} \bar{\partial}_{X} Z\right)+\left(\bar{\partial}_{X}-\bar{\partial}_{M}\right) F
\end{aligned}
$$

By Newton's method we would take $F=-P \bar{\partial}_{X} Z$. The integrability condition gives $\bar{\partial}_{M} \bar{\partial}_{X}=\left(\bar{\partial}_{M}-\bar{\partial}_{X}\right) \bar{\partial}_{X}$. The coefficients of $\bar{\partial}_{X}-\bar{\partial}_{M}$ are dominated by $\left\|\bar{\partial}_{X} Z\right\|$, by (1.8), (1.9). Thus, formally we would have $\left\|\bar{\partial}_{X} Z_{*}\right\| \leq\left\|\bar{\partial}_{X} Z\right\|^{2}$. However, the

operator $P$ may not fully regain the derivative lost in applying $\bar{\partial}_{X}$ to $Z$. Thus we introduce the smoothing operator $S_{t}$ of section 7 , and set

$$
F=-S_{t} P \bar{\partial}_{X} Z
$$

Then

$$
\bar{\partial}_{X} Z_{*}=\bar{\partial}_{M}\left(I-S_{t}\right) P \bar{\partial}_{X} Z+Q \bar{\partial}_{M} \bar{\partial}_{X} Z+\left(\bar{\partial}_{X}-\bar{\partial}_{M}\right) F
$$

This 3-term decomposition can be made to work [24]; however, its main deficiency seems to be that there is no smoothing in the $Q$ term. Thus, we introduce the commutator, applying $S_{t}$ to the coefficients of a differential form, and write

$$
\bar{\partial}_{X} Z_{*}=\left[\bar{\partial}_{M},\left(I-S_{t}\right)\right] P \bar{\partial}_{X} Z+\left(I-S_{t}\right) \bar{\partial}_{M} P \bar{\partial}_{X} Z+Q \bar{\partial}_{M} \bar{\partial}_{X} Z+\left(\bar{\partial}_{X}-\bar{\partial}_{M}\right) F
$$


Using the homotopy formula, $\bar{\partial}_{M} P \bar{\partial}_{X} Z=\bar{\partial}_{X} Z-Q \bar{\partial}_{M} \bar{\partial}_{X} Z$, a second time, in the second term, gives

$$
\bar{\partial}_{X} Z_{*}=I_{1}+I_{2}+I_{3}+I_{4}
$$

where

$$
\begin{aligned}
& I_{1}=\left(I-S_{t}\right) \bar{\partial}_{X} Z \quad ; \quad I_{2}=\left(\bar{\partial}_{M}-\bar{\partial}_{X}\right) S_{t} P \bar{\partial}_{X} Z ; \\
& I_{3}=\left[S_{t}, \bar{\partial}_{M}\right] P \bar{\partial}_{X} Z ; \quad I_{4}=S_{t} Q\left(\bar{\partial}_{M}-\bar{\partial}_{X}\right) \bar{\partial}_{X} Z .
\end{aligned}
$$

One may see that formally each term contains the product of 2 "small" factors, hence should be "smaller". The precise estimates will be given below.

\section{Renormalization of the embedding.}

While the change of section 3 will take an embedding with "small" error to one with "smaller" error, it tends to destroy the normalizations of lemma 2.1, which we use to set up and estimate the homotopy formula. In this section we give a precise procedure to restore these normalizations, while retaining a "smaller" error. We point out that this section represents a considerable simplification and improvement over the corresponding argument in [24], where third order normalization was used.

We now assume that $\left\|\bar{\partial}_{X} Z\right\|$ is "small", and (see (1.8),(1.9))

$$
\bar{\partial}_{X} Z(0)=0, \Leftrightarrow A_{\alpha}^{\bar{\beta}}(0)=0, B_{\alpha}(0)=0,
$$

which clearly holds for the initial embedding of section one. We shall renormalize $Z_{*}=Z+F, F$ given by (3.2), using the first order Taylor polynomial of $F$ at 0 ,

$$
\begin{aligned}
F\left(z^{\prime}, x^{n}\right) & =K_{0}+K_{\alpha} z^{\alpha}+K_{\bar{\alpha}} z^{\bar{\alpha}}+K_{n} x^{n}+F_{(2)}, \\
& \equiv K_{0}+\hat{K} \cdot x+F_{(2)}(x), \\
F_{(2)}\left(z^{\prime}, x^{n}\right) & =O(2),
\end{aligned}
$$

where each $K_{j}=\left(K_{j}^{\alpha}, K_{j}^{n}\right) \in \mathbf{C}^{n}$ is a constant vector, and "small". Notice that $X_{\bar{\alpha}} Z_{*}(0)=X_{\bar{\alpha}} F(0)=K_{\bar{\alpha}}$, so that $\left|K_{\bar{\alpha}}\right| \leq\left\|\bar{\partial}_{X} Z_{*}\right\|_{0}$ is actually a "smaller" quantity.

Now we replace $Z_{*}=Z+F$ by

$$
\begin{aligned}
Z_{*} & =Z+F+E=Z+\left(F_{(2)}-i K_{n} y^{n}\right), \\
E & =-K_{0}-K_{\alpha} z^{\alpha}-K_{n} z^{n}-K_{\bar{\alpha}} z^{\bar{\alpha}} .
\end{aligned}
$$

It follows that $\bar{\partial}_{X} Z_{*}(0)=0$, since $y^{n}=O(2)$; and $\left\|\bar{\partial}_{X} Z_{*}\right\|$ remains "smaller", since each $X_{\bar{\beta}} E=-K_{\alpha} X_{\bar{\beta}} z^{\alpha}-K_{n} X_{\bar{\beta}} z^{n}-K_{\bar{\beta}}$ is "smaller". We write (4.3) as

$$
\begin{aligned}
& z_{*}^{\alpha}=z^{\alpha}+f_{(2)}^{\alpha}, \quad f_{(2)}^{\alpha}=F_{(2)}^{\alpha}-i K_{n}^{\alpha} y^{n} \\
& z_{*}^{n}=z^{n}+F_{(2)}^{n}-i K_{n}^{n} y^{n} .
\end{aligned}
$$


With $K_{n}^{n}=K_{n}^{\prime n}+i K_{n}^{\prime \prime} n$, the second equation becomes

$$
\begin{aligned}
x_{*}^{n} & =x^{n}+f_{(2)}^{n}, \quad f_{(2)}^{n}=\operatorname{Re}\left(F_{(2)}^{n}\right)+K_{n}^{\prime \prime n} y^{n}, \\
y_{*}^{n} & =y^{n}+\operatorname{Im}\left(F_{(2)}^{n}\right)-K_{n}^{\prime n} y^{n} \\
& =\left|z^{\prime}\right|^{2}+h+\hat{h}, \\
\hat{h} & =\operatorname{Im}\left(F_{(2)}^{n}\right)-K_{n}^{\prime \prime n} y^{n} .
\end{aligned}
$$

The first equations in (4.5) and (4.6) define a map $f$, which is the projection of the map $Z+F+E$ to the real hyperplane $y^{n}=0$. It is a local diffeomorphism of neighborhoods of 0 in $\mathbf{R}^{2 n-1}$. We let $g$ be its inverse. Then

$$
f=I+f_{(2)}, \quad f^{-1}=g=I+g_{(2)},
$$

where $f_{(2)}=O(2), g_{(2)}=O(2)$. We define the new normalized embedding $Z_{1}$ and new real hypersurface $M_{1}$, the image of $Z_{1}$, by

$$
\begin{aligned}
Z_{1} & =Z_{*} \circ g=(Z+F+E) \circ g=\left(z^{\prime}, z_{1}^{n}\right), \\
z_{1}^{n} & =x^{n}+i y_{1}^{n}\left(z^{\prime}, x^{n}\right), \\
M_{1} & =\left\{r_{1}=0\right\}, \quad r_{1}=-y^{n}+\left|z^{\prime}\right|^{2}+h_{1}\left(z^{\prime}, x^{n}\right) .
\end{aligned}
$$

We have

$$
h_{1}\left(z^{\prime}, x^{n}\right)=(h+\hat{h}) \circ g+\delta_{\alpha \bar{\beta}}\left(z^{\alpha} g_{(2)}^{\bar{\beta}}+g_{(2)}^{\alpha} z^{\bar{\beta}}+g_{(2)}^{\alpha} g_{(2)}^{\bar{\beta}}\right) \text {, }
$$

or

$$
h_{1}-h=(h \circ g-h)+\hat{h} \circ g+\delta_{\alpha \bar{\beta}}\left(z^{\alpha} g_{(2)}^{\bar{\beta}}+g_{(2)}^{\alpha} z^{\bar{\beta}}+g_{(2)}^{\alpha} g_{(2)}^{\bar{\beta}}\right) \text {. }
$$

To determine the new adapted frame field $X_{\alpha}^{1}$ for $Z_{1}$, we think of $f$ as mapping our given $\mathrm{CR}$ structure to an equivalent one. To achieve $\delta_{\alpha}^{\beta}=X_{\alpha}^{1} z_{1}^{\beta}$, we define a matrix of functions $C_{\alpha}^{\beta} X_{\beta}$ by the following. (Note that $f_{*}(V)_{x}[h]=V_{g(x)}[h \circ f]$, and $g \circ f=i d$.)

$$
\begin{aligned}
X_{\alpha}^{1} & =f_{*}\left(C_{\alpha}^{\beta} X_{\beta}\right), \\
\delta_{\alpha}^{\beta} & =C_{\alpha}^{\gamma} X_{\gamma}[Z+F+E]^{\beta}=C_{\alpha}^{\gamma}\left(\delta_{\gamma}^{\beta}+X_{\gamma} f_{(2)}^{\beta}\right), \\
C_{\alpha}^{\beta} & =\delta_{\alpha}^{\beta}-X_{\alpha} f_{(2)}^{\gamma}\left(\delta_{\gamma}^{\beta}+X_{\gamma} f_{(2)}^{\beta}\right)^{-1},
\end{aligned}
$$

where $(\cdot)^{-1}$ indicates matrix inverse. This relies on the smallness of $X_{\alpha} f_{(2)}^{\beta}$ near 0 . We also have, using $g_{*} f_{*}=I$,

$$
\begin{aligned}
\bar{\partial}_{X^{1}} Z_{1} \equiv & \left\{X_{\bar{\alpha}}^{1} Z_{1}\right\}=\left\{\left(g_{*}\left(X_{\bar{\alpha}}^{1}\right)[Z+F+E]\right) \circ g\right\} \\
= & \left\{\left(C_{\bar{\alpha}} W_{\bar{\beta}}\right) \circ g\right\} \\
W_{\bar{\beta}}= & X_{\bar{\beta}}[Z+F]-X_{\bar{\beta}}[Z+F](0)+ \\
& -\partial_{\alpha} F(0) X_{\bar{\beta}} z^{\alpha}-\partial_{x^{n}} F(0) X_{\bar{\beta}} z^{n} .
\end{aligned}
$$


So far we have considered our maps $Z$ and $Z_{1}$ on the level of germs. As such, we have two well defined functional relations,

$$
Z_{1}=\mathcal{H}(Z, F), \quad \bar{\partial}_{X^{1}} Z_{1}=\mathcal{G}\left(Z, F, \bar{\partial}_{X} Z, D F\right) .
$$

Here the operator $\mathcal{H}$ is zero-th order, but involves composition with the inverse of the projected map $f$. The operator $\mathcal{G}$ is a first order differential operator also involving the composition.

To compare the actual domains of definition of $Z$ and $Z_{1}$, we must consider the inverse mapping theorem more carefully. We use the notation $\|\cdot\|_{\rho, k}=\|\cdot\|_{C^{k}\left(D_{\rho}\right)}$ of the next section.

Lemma 4.1. Let $D, h, \gamma_{0}, \rho_{0}, D_{\rho_{0}}$, and $0<\sigma<1$ be as in lemma 2.1, and let $\rho \leq \rho_{0}$. Suppose that $f=I+f_{(2)}$ is a smooth map from $D_{\rho}$ to $\mathbf{R}^{2 n-1}$, with

$$
f_{(2)}=O\left(|x|^{2}\right), \quad\left\|f_{(2)}\right\|_{\rho, 1} \leq \sigma / 5 .
$$

Then $f$ maps a compact smooth neighborhood $U_{\rho} \subseteq D_{\rho}$ of 0 , diffeomorphically onto $D_{\rho(1-\sigma)}$. The inverse map $g=f^{-1}$ has the form $g=I+g_{(2)}$, where

$$
g_{(2)}=O\left(|x|^{2}\right), \quad\left\|g_{(2)}\right\|_{\rho(1-\sigma), a} \leq c_{a}\left\|f_{(2)}\right\|_{\rho, a},
$$

for $0 \leq a \leq 2$, where the constant $c_{a}$ is independent of the domain and map.

Proof. The proof follows a standard argument for the inverse function theorem. We fix $x_{*} \in D_{\rho(1-\sigma)}$ and apply the contraction mapping principle to $x \mapsto w(x)=$ $x_{*}-f_{(2)}(x)$, on the domain $D_{\rho}$. Suppose $\left\|f_{(2)}\right\|_{\rho, 1} \leq \gamma \sigma$, with $\gamma>0$ to be chosen. By the convexity of $D_{\rho}$ and the mean value theorem on the segment $[0, x]$, we get $\left|f_{(2)}(x)\right| \leq\left\|f_{(2)}\right\|_{\rho, 1}|x|$. Then $\left|w(x)-x_{*}\right| \leq \gamma \sigma|x| \leq \gamma \sigma \sqrt{2} \rho$, by (2.3), for $x \in D_{\rho}$. By (2.6) this is bounded by $\sqrt{2} \gamma \hat{c} \operatorname{dist}\left(\partial D_{\rho_{0}(1-\sigma)}, \partial D_{\rho_{0}}\right)$. But $\sqrt{2} \hat{c} \leq 2\left(2+\gamma_{0}\right) \leq 5$ Thus $w$ maps $D_{\rho}$ into itself, if $\gamma=1 / 5$. Similarly for $x_{0}, x_{1}$ in $D_{\rho},\left|w\left(x_{1}\right)-w\left(x_{0}\right)\right| \leq$ $\gamma \sigma\left|x_{1}-x_{0}\right|$, so $w$ is a contraction there. It has a unique fixed point $x=g\left(x_{*}\right)$, $f\left(g\left(x_{*}\right)\right)=x_{*}$. The map $g$ is just as smooth as $f$, by the standard argument.

Since $f(0)=0, d f(0)=I$, we have $g(0)=0$, and by the chain rule, $d g(0)=I$. Thus $g=I+g_{(2)}, g_{(2)}=O(2)$, and the jacobian matrices further satisfy

$$
d g_{(2)}(x)=\Phi \circ d f_{(2)} \circ g(x), \Phi(W)=-W(I+W)^{-1} .
$$

Since $g_{(2)}(x)$ vanishes for $x=0$, we can bound it in terms of its first derivatives, as above. By (4.14) $\left|d f_{(2)}\right| \leq 1 / 2$, so (4.16) gives (4.15) for $a=1$.

For the Hölder continuity, let $0<\alpha<1$. We estimate the two compositions $u=\Phi \circ d f_{(2)}, v=u \circ g$ differently,

$$
\begin{aligned}
&\left|v\left(x_{1}\right)-v\left(x_{0}\right)\right| \leq H_{\alpha}(u)\left|g\left(x_{1}\right)-g\left(x_{0}\right)\right|^{\alpha} \leq H_{\alpha}(u)\left(1+\left\|g_{(2)}\right\|_{1}\right)^{\alpha}\left|x_{1}-x_{0}\right|^{\alpha}, \\
&\left|u\left(x_{1}\right)-u\left(x_{0}\right)\right| \leq\|\Phi\|_{1}\left|d f_{(2)}\left(x_{1}\right)-d f_{(2)}\left(x_{0}\right)\right| \leq\|\Phi\|_{1} H_{\alpha}\left(d f_{(2)}\right)\left|x_{1}-x_{0}\right|^{\alpha} .
\end{aligned}
$$


Combining gives (4.15) for $0<a<2$.

Finally, taking first partial derivatives in (4.16) and estimating gives (4.15) for $a=2$, in view of (4.14).

Next we assume that $f(4.7)$ is the projection of $Z+F+E$, and that $h_{1}$ is given by (4.9). We want to compare two of the domains $D_{\rho_{1}}\left(h_{1}\right)$ and $D_{\rho}(h)$.

Lemma 4.2. There is a constant $\gamma_{1}>0$ such that the following holds. Let $D, h$, $\gamma_{0}, \rho_{0}, D_{\rho_{0}}$, and $\sigma$ be as in lemma 4.1, with $0<\rho_{0} \leq 1$ and $0<\sigma<1 / 2$. Let $\rho \leq \rho_{0}$, and $f$ and $h_{1}$ be as above. If the map $F$ satisfies

$$
\|F\|_{\rho, 1} \leq \gamma_{1} \rho \sigma
$$

then lemma 4.1 holds, and the functions $g, Z_{1}$, and $h_{1}$ are defined on a neighborhood of $D_{\rho(1-\sigma)} \equiv D_{\rho(1-\sigma)}(h)$, and

$$
D_{\rho(1-2 \sigma)}\left(h_{1}\right) \subseteq D_{\rho(1-\sigma)}(h) .
$$

Proof. For the proof note that (4.5), (4.6) give

$$
\begin{aligned}
\left\|f_{(2)}\right\|_{\rho, 1} & \leq\left\|F_{(2)}\right\|_{\rho, 1}+|d F(0)|\left\|y^{n}\right\|_{\rho, 1} \\
& \leq 3\|F\|_{\rho, 1}+|d F(0)|\left(3 \sqrt{2} \rho+\|h\|_{\rho, 1}\right) \\
& \leq\|F\|_{\rho, 1}(3+3 \sqrt{2}+1 / 2) \leq 8\|F\|_{\rho, 1} .
\end{aligned}
$$

Thus, if we choose $\gamma_{1}<1 / 45$, then (4.14) and hence lemma 4.1 hold. We apply (4.10) and the Schwarz inequality to get

$$
\begin{gathered}
\psi(x)-\psi_{1}(x)=h(x)-h_{1}(x) \leq \\
|h(g(x))-h(x)|+|\hat{h}(g(x))|+\left(2|x|+\left|g_{(2)}(x)\right|\right)\left|g_{(2)}(x)\right| .
\end{gathered}
$$

We shall bound the first term on the right in (4.20) by the third term, as in the proof of lemma 2.1. For $x \in D_{\rho(1-\sigma)}(h)$, the segment $[x, g(x)]$ lies in $D_{\rho}(h)$. Thus we have

$$
\begin{aligned}
g_{t}(x) & =x+\operatorname{tg}_{(2)}(x)(\text { def. }), \\
h(g(x))-h(x) & =\int_{0}^{1} \partial_{j} h\left(g_{t}(x)\right) g_{(2)}^{j}(x) d t, \\
\left|\partial_{j} h\left(g_{t}(x)\right)\right| & \leq\|h\|_{\rho, 2}\left(|x|+\left|g_{(2)}(x)\right|\right), \\
|h(g(x))-h(x)| & \leq \gamma_{0}\left(|x|+\left|g_{(2)}(x)\right|\right)\left|g_{(2)}(x)\right| .
\end{aligned}
$$

To estimate the first and third terms on the right of (4.20), we note that $2|x|+$ $\left|g_{(2)}(x)\right| \leq 3|x|+|g(x)| \leq \sqrt{2}(3 \rho+\rho) \leq 4 \sqrt{2}$. Also, by (4.15), (4.19)

$$
\left|g_{(2)}(x)\right| \leq\left\|g_{(2)}\right\|_{\rho(1-\sigma), 1}|x| \leq 9 c_{1}\|F\|_{\rho, 1}|x| \leq 9 c_{1} \sqrt{2} \rho\|F\|_{\rho, 1} .
$$


For the second term, note that for $x \in D_{\rho}(h),(4.6)$ gives

$$
\begin{aligned}
|\hat{h}(x)| & \leq\left\|F_{(2)}\right\|_{\rho, 1}|x|+|d F(0)|\left(\left|z^{\prime}\right|^{2}+\left|h\left(z^{\prime}, x^{n}\right)\right|\right) \\
& \leq\|F\|_{\rho, 1}\left(2+\sqrt{2} \rho+\|h\|_{\rho, 1}\right)|x| \\
& \leq 5\|F\|_{\rho, 1}|x|
\end{aligned}
$$

For $x \in D_{\rho(1-\sigma)}(h)$, we have $g(x) \in D_{\rho}(h)$, so this gives

$$
|\hat{h}(g(x))| \leq 5 \sqrt{2} \rho\|F\|_{\rho, 1} .
$$

Combining the three terms and using (4.17) gives, for $x \in D_{\rho(1-\sigma)}(h)$,

$$
\psi(x)-\psi_{1}(x) \leq \tilde{c} \rho\|F\|_{\rho, 1} \leq \tilde{c} \gamma_{1} \rho^{2} \sigma
$$

for an absolute constant $\tilde{c}$.

We want to show that $\psi(x) \leq \rho^{2}(1-\sigma)^{2}$, if $\psi_{1}(x) \leq \rho^{2}(1-2 \sigma)^{2}$. This comes down to $3 \sigma+\tilde{c} \gamma_{1} \leq 2$. With $\sigma<1 / 2$, we require $\tilde{c} \gamma_{1}<1 / 2$. This proves the lemma.

By combining the results of sections 3 and 4 , we get the main step, $Z \mapsto Z_{1}$, in our iteration procedure. Basically, we shall need to control the new approximate embedding $Z_{1}$, or equivalently $h_{1}$, and the new error $\bar{\partial}_{X_{1}} Z_{1}$. As lemma 4.2 indicates, the control of $F(3.2)$ will be central to this process. This is begun in the next two sections.

\section{$5 \quad$ Hölder estimates.}

First we recall some basic results for standard Hölder norms $\|u\|_{a}=\|u\|_{C^{a}\left(D_{\rho}\right)}$, $0 \leq a<\infty$, for functions $u$ on a smooth, bounded, strictly convex domain $D_{\rho}$, with in-radius comparable to $\rho$,

$$
B\left(\epsilon_{1} \rho\right) \subseteq D_{\rho} \subseteq B\left(\epsilon_{2} \rho\right) \subset \mathbf{R}^{n}, 0<\epsilon_{1}<\epsilon_{2}
$$

For reference, see the appendices of [10] or [6], which we follow with some adaptation. Next we take $D_{\rho}=D_{\rho}(h)$, setting $\|u\|_{\rho, a}=\|u\|_{a}$, and make estimates for the new function $h_{1}$. From now on we shall assume $0<\rho \leq 1$.

Perhaps most basic is the interpolation estimate, where $a<c<b, 0<\lambda<1$, $c=\lambda a+(1-\lambda) b$

$$
\|u\|_{c} \leq c_{a} \rho^{-c}\|u\|_{a}^{\lambda}\|u\|_{b}^{1-\lambda} .
$$


From this one derives the convexity estimate, for functions $u, v$ on perhaps different domains $D_{\rho}, D_{\tau}$,

$$
\|u\|_{a}\|v\|_{b} \leq c_{a, b} \rho^{-a} \tau^{-b}\left(\|u\|_{a_{1}}\|v\|_{b_{1}}+\|u\|_{a_{2}}\|v\|_{b_{2}}\right),
$$

where $(a, b)=\lambda\left(a_{1}, b_{1}\right)+(1-\lambda)\left(a_{2}, b_{2}\right), 0<\lambda<1$.

The product-rule estimate for $u, v$ on $D_{\rho}$ is

$$
\|u v\|_{a} \leq c_{a} \rho^{-a}\left(\|u\|_{a}\|v\|_{0}+\|u\|_{0}\|v\|_{a}\right) .
$$

These estimates can be derived as in [10], with constants independent of $\rho$, but using scale invariant norms, and then passing back to standard norms. (To get "scale invariant" norms, multiply the sup norm of the $j$-th order derivatives by $\rho^{j}$, $0<j \leq k$, and the $\alpha$-Hölder ratio of the $k$-th order derivatives by $\rho^{k+\alpha}$, and add together.)

As indicated in [10], there are two ways to make a chain-rule estimate. Our first is somewhat weaker than the corresponding one in [10], but in a more precise form. For maps $g: D_{\rho} \rightarrow D_{\tau}$ and $u: D_{\tau} \rightarrow \mathbf{R}^{N}, 0<\rho, \tau \leq 1$, we have

$$
\begin{aligned}
\|u \circ g\|_{a} & \leq K_{a}\left(\|u\|_{a}+\|u\|_{1}\|g\|_{a}\right) \\
K_{a} & =c_{a} \tau^{-2 a} \rho^{-a^{2}}\left(1+\|g\|_{1}\right)^{2 a}
\end{aligned}
$$

In case $D_{\tau}=B(1 / 2)$ and $u$ is a fixed rational or analytic function on $B(1)$, (specifically the matrix inverse $\left.u(W)=(I+W)^{-1}\right)$, we have

$$
\begin{aligned}
\|u \circ g\|_{a} & \leq K_{a}\|g\|_{a}, \\
K_{a} & =c_{a} \rho^{-a}\left(1+\|g\|_{0}\right)^{a-1} .
\end{aligned}
$$

We augment lemma 4.1 with the following. It is a refinement of the inverse mapping estimate of [10]. See appendix A of [6].

Lemma 5.1. With the hypothesis of lemma 4.1, we also have

$$
\left\|g_{(2)}\right\|_{\rho(1-\sigma), a} \leq c_{a} \rho^{-4(a+2)}\left\|f_{(2)}\right\|_{\rho, a},
$$

for $0 \leq a<\infty$. For $0 \leq a \leq 2$ there is no $\rho$-factor, by (4.15).

In lemma $4.2, f_{(2)}$ is given by (4.5), (4.6) and (4.2), (1.5), and we assume $\|h\|_{\rho, 2} \leq$ $\gamma_{0}<1 / 2$, and $\|F\|_{\rho, 1} \leq \gamma_{1} \rho \sigma<1 / 2$. Then as in (4.19) we have

$$
\left\|f_{(2)}\right\|_{\rho, a} \leq c_{a}\left(\|F\|_{\rho, a}+\|F\|_{\rho, 1}\|h\|_{\rho, a}\right), \quad 1 \leq a<\infty .
$$


Lemma 5.2. Let the hypotheses be as in lemma 4.2. For $\rho_{1}=\rho(1-\sigma)$, we have (the domains are $D_{\rho_{1}}(h)$ and $D_{\rho}(h)$ )

$$
\begin{aligned}
\left\|h_{1}\right\|_{\rho_{1}, a} & \leq c_{a} \rho^{-a_{2}}\left(\|h\|_{\rho, a}+\|F\|_{\rho, a}\right), \\
\left\|h_{1}-h\right\|_{\rho_{1}, a} & \leq c_{a} \rho^{-a_{2}}\left(\|F\|_{\rho, 1}\|h\|_{\rho, a+1}+\|F\|_{\rho, a}\right),
\end{aligned}
$$

for $1 \leq a<\infty$, where $a_{2}$ is quadratic in $a$.

Proof. To prove (5.9), we use the chain-rule and product-rule estimates in (4.9) to get

$$
\begin{aligned}
\left\|h_{1}\right\|_{\rho_{1}, a} \leq & c_{a} \rho^{-a_{2}}\left(\|h+\hat{h}\|_{\rho, a}+\|h+\hat{h}\|_{\rho, 1}\left\|g_{(2)}\right\|_{\rho_{1}, a}\right)+ \\
& +\left(2+c_{a} \rho^{-a}\left\|g_{(2)}\right\|_{\rho_{1}, 0}\right)\left\|g_{(2)}\right\|_{\rho_{1}, a} .
\end{aligned}
$$

From (4.6) and (1.4), (1.5) we get

$$
\|\hat{h}\|_{\rho, a} \leq c_{a}\left(\|F\|_{\rho, a}+\|F\|_{\rho, 1}\|h\|_{\rho, a}\right) .
$$

Now we apply (5.7), (5.8) and use $\|F\|_{\rho, 1} \leq 1$. After simplifying, we get (5.9) (with $\left.a_{2}=a^{2}+7 a+8\right)$.

To prove (5.10) we estimate the first term in (4.10) using calculus, as in (4.21)(4.22), and the product rule estimate, getting (with norms over the appropriate domains)

$$
\begin{aligned}
\|h \circ g-h\|_{a} & \leq c_{a} \rho^{-a} \int_{0}^{1}\left\{\left\|D h \circ g_{t}\right\|_{a}\left\|g_{(2)}\right\|_{0}+\left\|D h \circ g_{t}\right\|_{0}\left\|g_{(2)}\right\|_{a}\right\} \\
& \leq c_{a} \rho^{-a-a^{2}}\left\{\|h\|_{a+1}\left\|g_{(2)}\right\|_{0}+\|h\|_{2}\left\|g_{(2)}\right\|_{a}\right\}
\end{aligned}
$$

and we use $\|h\|_{2} \leq 1$. The other terms are treated as in the proof of (5.9), with $h$ dropped from the expression $(h+\hat{h})$. After simplifying, we get (5.10).

\section{A general estimate for the new error.}

We derive a "general" estimate for the new error $\bar{\partial}_{X^{1}} Z_{1}$ in terms of the previous embedding and the function $F$. This will be specialized to give coarse and fine estimates for the new error. We assume that our vector fields $X_{\alpha}$ are in the Hölder class $C^{m}, m \in \mathbf{R}, m \geq 2$.

We first estimate the $X_{\alpha}$ derivative of a function $u$. This is straight forward using the product-rule estimate twice, and (1.8), (1.9). 
Lemma 6.1. For $0 \leq a \leq m$, we have, on a fixed domain, (6.1)

$$
\begin{aligned}
\left\|X_{\bar{\alpha}} u\right\|_{a} & \leq K_{a}^{\prime}\left(\|u\|_{a+1}+\left(\left\|\bar{\partial}_{X} Z\right\|_{a}+\|h\|_{a+1}\right)\|u\|_{1}\right) \\
\left\|X_{\bar{\alpha}} u-Y_{\bar{\alpha}} u\right\|_{a} & \leq K_{a}^{\prime}\left(\left\|\bar{\partial}_{X} Z\right\|_{0}\|u\|_{a+1}+\left(\left\|\bar{\partial}_{X} Z\right\|_{0}\|h\|_{a+1}+\left\|\bar{\partial}_{X} Z\right\|_{a}\right)\|u\|_{1}\right), \\
K_{a}^{\prime} & =c_{a} \rho^{-2 a}\left(1+\left\|\bar{\partial}_{X} Z\right\|_{0}\right) .
\end{aligned}
$$

The first estimate of the lemma is applied to get

$$
\begin{aligned}
\left\|X_{\alpha} f_{(2)}^{\beta}\right\|_{\rho, 0} & \leq c_{0}\left(1+\left\|\bar{\partial}_{X} Z\right\|_{\rho, 0}\right)^{2}\left\|f_{(2)}\right\|_{\rho, 1} \\
& \leq 3 c_{0}\left\|f_{(2)}\right\|_{\rho, 1},
\end{aligned}
$$

if we assume $\left\|\bar{\partial}_{X} Z\right\|_{\rho, 0} \leq 1 / 2$, say. Then, if $\left\|f_{(2)}\right\|_{\rho, 1}$ is sufficiently small, the inverse matrix in (4.11) will exist and (4.12) will be valid.

We have the following general estimate.

Lemma 6.2. Let the hypotheses of lemma 4.2 hold, $\left\|\bar{\partial}_{X} Z\right\|_{\rho, 1} \leq 1 / 2$, and $\rho_{1}=$ $\rho(1-\sigma)$. Then

$$
\begin{aligned}
\left\|\bar{\partial}_{X^{1}} Z_{1}\right\|_{\rho_{1}, a} \leq & c_{a} \rho^{-a_{2}}\left\{\left\|\bar{\partial}_{X}(Z+F)\right\|_{\rho, a}+\|F\|_{\rho, 1}\left\|\bar{\partial}_{X} Z\right\|_{\rho, a}+\right. \\
& +\left(\left\|\bar{\partial}_{X} Z\right\|_{\rho, 0}+\|F\|_{\rho, 1}\right)\left(\|F\|_{\rho, a+1}+\|F\|_{\rho, 1}\|h\|_{\rho, a+1}\right)+ \\
& \left.+\left\|\bar{\partial}_{X} Z\right\|_{\rho, 1}\left(\|F\|_{\rho, a}+\|F\|_{\rho, 1}\|h\|_{\rho, a}\right)\right\}
\end{aligned}
$$

for $1 \leq a \leq m$.

Proof. We must estimate (4.12), which we abbreviate as

$$
\bar{\partial}_{X^{1}} Z_{1}=(\overline{C W}) \circ g, \quad C=I-X f_{(2)}\left(I+X f_{(2)}\right)^{-1} .
$$

For the following 4 estimates, we use, respectively, chain rule (5.5) and lemma 5.1; the product rule and chain rule (5.6); the product rule; and lemma 6.1.

$$
\begin{aligned}
\left\|\bar{\partial}_{X^{1}} Z_{1}\right\|_{\rho_{1}, a} & \leq c_{a} \rho^{-a_{2}}\left(\|C W\|_{a}+\|C W\|_{1}\left\|f_{(2)}\right\|_{a}\right) ; \\
\|C\|_{a} & \leq c_{a} \rho^{-2 a}\left(1+\left\|X f_{(2)}\right\|_{a}\right) \\
\|C W\|_{a} & \leq c_{a} \rho^{-3 a}\left(\|W\|_{a}+\|W\|_{0}\left\|X f_{(2)}\right\|_{a}\right), \\
\left\|X f_{(2)}\right\|_{a} & \leq c_{a} \rho^{-2 a}\left(\left\|f_{(2)}\right\|_{a+1}+\left\|f_{(2)}\right\|_{1}\left(\left\|\bar{\partial}_{X} Z\right\|_{a}+\|h\|_{a+1}\right)\right) .
\end{aligned}
$$

In combining and simplifying, we note that under our hypotheses, $1+\left\|f_{(2)}\right\|_{1} \leq 2$, $\left\|\bar{\partial}_{X} Z\right\|_{1}+\|h\|_{2} \leq 2$, and

$$
\left\|f_{(2)}\right\|_{2}\left\|f_{(2)}\right\|_{a} \leq 2 c_{a} \rho^{-2-a}\left\|f_{(2)}\right\|_{1}\left\|f_{(2)}\right\|_{a+1},
$$

by the convexity estimate (5.3). This leads to

$$
\begin{aligned}
\left\|\bar{\partial}_{X^{1}} Z_{1}\right\|_{\rho_{1}, a} \leq & c_{a} \rho^{-a_{2}}\left\{\|W\|_{a}+\|W\|_{1}\left\|f_{(2)}\right\|_{a}+\right. \\
& \left.+\|W\|_{0}\left(\left\|f_{(2)}\right\|_{a+1}+\left\|f_{(2)}\right\|_{1}\left(\left\|\bar{\partial}_{X} Z\right\|_{a}+\|h\|_{a+1}\right)\right)\right\} .
\end{aligned}
$$


From (4.12) we get

$$
\|W\|_{a} \leq 2\left\|\bar{\partial}_{X}(Z+F)\right\|_{a}+\|F\|_{1}\left\|\bar{\partial}_{X} Z\right\|_{a} .
$$

We substitute this for the $W$-terms and use the convexity estimate (5.3) for $\|F\|_{2}\|F\|_{a}$ and $\|F\|_{2}\|h\|_{a}$ as above. This gives the lemma.

\section{Estimates for $S_{t}, P$, and $Q$.}

Here we summarize some results on a standard smoothing operator $S_{t}$, and give an estimate for the Friedrichs commutator. Then we recall some necessary results from [6] estimating the homotopy operators $P$ and $Q$.

By lemma 2.1 we may define a standard smoothing operator $S_{t}: C\left(D_{\rho}\right) \rightarrow$ $C^{\infty}\left(D_{\rho_{1}}\right), \rho_{1}=\rho(1-\sigma), 0<t<\hat{c}^{-1} \rho \sigma, \hat{c}=3 \sqrt{2}$

$$
S_{t} u(x)=\int_{|x-y|<\rho \sigma} u(y) \chi_{t}(x-y) d y=\int_{|z|<1} \chi(z) u(x-t z) d z,
$$

where spt $\chi \subset \subset\{|z|<1\}, \int \chi(z) d z=1$, and $\int z^{I} \chi(z) d z=0$ for $0<|I|<2 m$, say.

We have the basic estimates [17], [24]

$$
\begin{aligned}
\left\|S_{t} u\right\|_{\rho_{1}, a} & \leq c_{a} \rho^{-a} t^{b-a}\|u\|_{\rho, b} \quad, \quad 0 \leq b \leq a<\infty \\
\left\|\left(I-S_{t}\right) u\right\|_{\rho_{1}, a} & \leq c_{m} \rho^{-a} t^{b-a}\|u\|_{\rho, b} \quad, \quad 0 \leq a \leq b<2 m .
\end{aligned}
$$

For $b-a \in \mathbf{Z}$ these hold without the $\rho$-factors. For $b-a \in \mathbf{R}$ they follow by means of the interpolation estimate (5.2).

The commutator $\left[S_{t}, \bar{\partial}_{M}\right]$ is equivalent to all $\left[S_{t}, Y_{\bar{\alpha}}\right]=\left[S_{t}, w\right] \partial_{x^{n}}$, where $w=$ $-r_{\bar{\alpha}} / 2 r_{\bar{n}}$.

Lemma 7.1. For $k \in \mathbf{Z}^{+}, 0 \leq \alpha \leq 1,0<t<\hat{c}^{-1} \rho \sigma$, we have

$$
\begin{aligned}
\left\|\left[S_{t}, \bar{\partial}_{M}\right] u\right\|_{\rho_{1}, k} & \leq c_{m} \rho^{-k-\alpha} t^{\alpha}\left\{\|r\|_{\rho, 2}\|u\|_{\rho, k+\alpha}+\|r\|_{\rho, k+2}\|u\|_{\rho, \alpha}\right\} \\
\left\|\left[S_{t}, \bar{\partial}_{M}\right] u\right\|_{\rho_{1}, k+\alpha} & \leq c_{m} \rho^{-k-\alpha}\left\{\|r\|_{\rho, 2}\|u\|_{\rho, k+\alpha}+\|r\|_{\rho, k+2+\alpha}\|u\|_{\rho, 0}\right\} .
\end{aligned}
$$

Proof. We set $v(x)=\left[S_{t}, w\right] \partial_{x^{n}} u(x)$, and integrate by parts to take the derivative $\partial_{x^{n}}$ off $u$. This gives

$$
\begin{aligned}
v(x) & =\int_{|z| \leq 1} R(x, z, t)(u(x-t z)-u(x)) d z, \\
R(x, z, t) & =\partial_{z^{n}} \chi(z) t^{-1}(w(x-t z)-w(x))-\chi(z) \partial_{x^{n}} w(x-t z) .
\end{aligned}
$$

For the sup norm and Holder ratio, we readily derive

$$
\begin{aligned}
\|v\|_{0} & \leq c_{0}\left\|w_{x}\right\|_{0} t^{\alpha} H_{\alpha}(u), \\
H_{\alpha}(v) & \leq c_{\alpha}\left(\left\|w_{x}\right\|_{0} H_{\alpha}(u)+H_{\alpha}\left(w_{x}\right)\|u\|_{0}\right) .
\end{aligned}
$$


We take a first order $x$-derivative $D$ by means of

$$
D\left[S_{t}, w\right] \partial_{x^{n}} u=\left[S_{t}, D w\right] \partial_{x^{n}} u(x)+\left[S_{t}, w\right] \partial_{x^{n}} D u(x) .
$$

We take $k$ such derivatives and apply (7.6) and the convexity estimate (5.3). This gives the lemma.

In [6] we have derived the following estimates, coarse and fine, for the homotopy formula.

Lemma 7.2. Let the domain $D_{\rho}$ and the corresponding hypersurface $M_{\rho}$ be as in lemma 2.1. Then the operators $P$ and $Q$ in the homotopy formula (2.13) for $M_{\rho}$ satisfy the following estimates.

$$
\|P \phi\|_{\rho_{1}, a} \leq K(a)\left\{\|\phi\|_{\rho, a}+\|h\|_{\rho, a+2}\|\phi\|_{\rho, 0}\right\}, \quad K(a)=c_{a}(\rho \sigma)^{-s(a)}
$$

where $\rho_{1}=\rho(1-\sigma)$, and $s(a)$ is some polynomial in $a, 0 \leq a<\infty$. Also, for $k \in \mathbf{Z}$, $0 \leq k, \alpha=1 / 2$, and $\beta=5 / 2$,

$$
\|P \phi\|_{\rho_{1}, k+\alpha} \leq K(k+\alpha)\left\{\left(1+\|h\|_{\rho, \beta}\right)\|\phi\|_{\rho, k}+\|h\|_{\rho, k+\beta}\|\phi\|_{\rho, 0}\right\} .
$$

For the special case of forms $\phi$ of type $(0,1), k=0$, and $\alpha=1 / 2$, we have

$$
\|P \phi\|_{\rho_{1}, 1 / 2} \leq c_{1} \rho^{-3 / 2} \sigma^{-2 n}\|\phi\|_{\rho, 0} .
$$

In what follows we shall increase $c_{a}$ and $s(a)$ a finite number of times, but keep the same notation $K(a)$.

\section{Estimates for $F$.}

In sections 5 and 6 we have derived estimates for $h_{1}$ and $\bar{\partial}_{X^{1}} Z_{1}$ in terms of $h, \bar{\partial}_{X} Z$, and $F$. Now we use the results of section 7 to estimate $F$ and to develop these results further.

Our basic iteration step will be the following. Given $Z$ and $\bar{\partial}_{X} Z$ on $D_{\rho}(h)$ as in lemma 2.1, we form $P \bar{\partial}_{X} Z$ on $D_{\rho}(h)$ and estimate it on $D_{\rho(1-\sigma)}(h), 0<\sigma<1 / 2$, using lemma 7.2. For $0<t \leq \hat{c}^{-1} \rho \sigma$, we define and estimate $F=-S_{t} P \bar{\partial}_{X} Z$ and the projected map $f=I+f_{(2)}$ on $D_{\rho(1-\sigma)^{2}}(h)$ using (7.2). Then lemma 4.2 gives $g=I+g_{(2)}, Z_{1}, h_{1}$ on $D_{\rho(1-\sigma)^{3}}(h)$, which can be estimated on $D_{\rho(1-\sigma)^{4}}(h)$. This last domain contains $D_{\rho(1-\sigma)^{3}(1-2 \sigma)}\left(h_{1}\right)$, which in turn contains $D_{\rho_{1}}\left(h_{1}\right), \rho_{1}=\rho(1-5 \sigma)$, $0<\sigma<1 / 5$, since $(1-\sigma)^{3}(1-2 \sigma) \geq(1-3 \sigma)(1-2 \sigma) \geq(1-5 \sigma)$. We may now take norms $\|\cdot\|_{\rho_{1}, a}$ over $D_{\rho_{1}}\left(h_{1}\right)$ on the left hand sides of the estimates. 
For convenience we put

$$
\tilde{\rho}(j)=\rho(1-\sigma)^{j}, 0<j \leq 4,
$$

relative to norms on the domains defined by the function $h$. From (7.2) and (7.10) we get

$$
\|F\|_{\tilde{\rho}(2), 1} \leq c_{1} \rho^{-5 / 2} \sigma^{-2 n} t^{-1 / 2}\left\|\bar{\partial}_{X} Z\right\|_{\rho, 0} .
$$

Thus the condition (4.17) in lemma 4.2 will hold if

$$
t^{-1 / 2}\left\|\bar{\partial}_{X} Z\right\|_{\rho, 1} \leq \gamma_{1} \rho^{7 / 2} \sigma^{2 n+1},
$$

where we have replaced $\gamma_{1}$ by a possibly smaller positive constant. This will also guarantee the condition in lemma 6.2 , as $t \leq 1$.

Combining (7.2) and (7.8) gives, with $b \leq a$ and $b \leq m$, and a possibly larger $K(a)$ of the same form $(7.8)$,

$$
\begin{aligned}
\|F\|_{\tilde{\rho}(2), a} & \leq c_{a} \rho^{-a} t^{b-a}\left\|P \bar{\partial}_{X} Z\right\|_{\rho, b} \\
& \leq K(a) t^{b-a}\left\{\left\|\bar{\partial}_{X} Z\right\|_{\rho, b}+\|h\|_{\rho, b+2}\left\|\bar{\partial}_{X} Z\right\|_{\rho, 0}\right\}, \\
\|F\|_{\tilde{\rho}(2), 1} & \leq K(1)\left(1+\|h\|_{\rho, 3}\right)\left\|\bar{\partial}_{X} Z\right\|_{\rho, 1} .
\end{aligned}
$$

We may instead use (7.2) and (7.9), with $b=l+(1 / 2) \leq a, l \in \mathbf{Z}, l \leq m$. This gives the following alternative estimate.

$$
\|F\|_{\tilde{\rho}(2), a} \leq K(a) t^{l+(1 / 2)-a}\left\{\left(1+\|h\|_{\rho, \beta}\right)\left\|\bar{\partial}_{X} Z\right\|_{\rho, l}+\|h\|_{\rho, l+\beta}\left\|\bar{\partial}_{X} Z\right\|_{\rho, 0}\right\} .
$$

For the map $f$ we have, using (5.8), (8.2), (8.4),

$$
\begin{aligned}
\left\|f_{(2)}\right\|_{\tilde{\rho}(2), a} \leq & K(a)\left\{t^{-1 / 2}\left\|\bar{\partial}_{X} Z\right\|_{\rho, 0}\|h\|_{\rho, a}+\right. \\
& \left.+t^{b-a}\left[\left\|\bar{\partial}_{X} Z\right\|_{\rho, b}+\|h\|_{\rho, b+2}\left\|\bar{\partial}_{X} Z\right\|_{\rho, 0}\right]\right\},
\end{aligned}
$$

where $b \leq a$, and we recopy (5.7) as

$$
\left\|g_{(2)}\right\|_{\rho_{1}, a} \leq\left\|g_{(2)}\right\|_{\tilde{\rho}(3), a} \leq c_{a} \rho^{-4(a+2)}\left\|f_{(2)}\right\|_{\tilde{\rho}(2), a}
$$

To refine the estimates for $h_{1}$, we now take $\rho_{1}=\rho(1-5 \sigma)$ on the left-hand sides in (5.9) and (5.10), and norms $\|\cdot\|_{\tilde{\rho}(2), *}$ on the right-hand sides. Using (8.2) and either (8.4) or (8.5), we get the following coarse and fine estimates for $h_{1}$.

Lemma 8.1. Relative to the above described domains, and $1 \leq a<\infty, b \leq a$, $b \leq m$, or $k \in \mathbf{Z}, k+(1 / 2) \leq a, k \leq m$, we have

$$
\begin{aligned}
\left\|h_{1}\right\|_{\rho_{1}, a} \leq & K(a)\left\{\|h\|_{\rho, a}+\right. \\
& \left.+t^{b-a}\left(\left\|\bar{\partial}_{X} Z\right\|_{\rho, b}+\|h\|_{\rho, b+2}\left\|\bar{\partial}_{X} Z\right\|_{\rho, 0}\right)\right\}, \\
\left\|h_{1}\right\|_{\rho_{1}, a} \leq & K(a)\left\{\|h\|_{\rho, a}+\right. \\
& \left.+t^{k+(1 / 2)-a}\left[\left(1+\|h\|_{\rho, \beta}\right)\left\|\bar{\partial}_{X} Z\right\|_{\rho, k}+\|h\|_{\rho, k+\beta}\left\|\bar{\partial}_{X} Z\right\|_{\rho, 0}\right]\right\},
\end{aligned}
$$




$$
\begin{aligned}
\left\|h_{1}-h\right\|_{\rho_{1}, a} \leq & K(a)\left\{t^{-1 / 2}\left\|\bar{\partial}_{X} Z\right\|_{\rho, 0}\|h\|_{\rho, a+1}+\right. \\
& \left.+t^{b-a}\left(\left\|\bar{\partial}_{X} Z\right\|_{\rho, b}+\|h\|_{\rho, b+2}\left\|\bar{\partial}_{X} Z\right\|_{\rho, 0}\right)\right\} .
\end{aligned}
$$

For a coarse estimate of the new error $\bar{\partial}_{X^{1}} Z_{1}$, we may simplify (6.3) to (8.11)

$$
\begin{aligned}
\left\|\bar{\partial}_{X^{1}} Z_{1}\right\|_{\rho_{1}, a} \leq & c_{a} \rho^{-a_{2}}\left\{\left\|\bar{\partial}_{X}(Z+F)\right\|_{\tilde{\rho}(2), a}+\|F\|_{\tilde{\rho}(2), 1}\left\|\bar{\partial}_{X} Z\right\|_{\rho, a}+\right. \\
& +\left(\left\|\bar{\partial}_{X} Z\right\|_{\rho, 1}+\|F\|_{\tilde{\rho}(2), 1}\left(\|F\|_{\tilde{\rho}(2), a+1}+\|F\|_{\tilde{\rho}(2), 1}\|h\|_{\rho, a+1}\right)\right\} .
\end{aligned}
$$

Using (8.4) with $b=a<a+1$ to estimate $\|F\|_{\tilde{\rho}(2), a+1}$ and with $b=a=1$ to estimate $\|F\|_{\tilde{\rho}(2), 1}$, and combining some constants gives the following estimate.

Lemma 8.2. Assuming the above and $1 \leq a \leq m$, we have

$$
\begin{aligned}
\left\|\bar{\partial}_{X^{1}} Z_{1}\right\|_{\rho_{1}, a} \leq & K(a+1)\left\{\left\|\bar{\partial}_{X}(Z+F)\right\|_{\tilde{\rho}(2), a}+\left(1+\|h\|_{\rho, 3}\right)^{2}\right. \\
& \left.\cdot t^{-1}\left\|\bar{\partial}_{X} Z\right\|_{\rho, 1}\left(\left\|\bar{\partial}_{X} Z\right\|_{\rho, a}+\left\|\bar{\partial}_{X} Z\right\|_{\rho, 1}\|h\|_{\rho, a+2}\right)\right\} .
\end{aligned}
$$

\section{Four-term estimates for $\bar{\partial}_{X}(Z+F)$.}

This is the central part of the estimates, and is based on the 4 term decomposition of section 3 . We follow the scheme for the domains set down at the beginning of the last section. We consider both $a$-norms and $k$-norms, $a \in \mathbf{R}, k \in \mathbf{Z}, 1 \leq a, k \leq m$. Also we use the notations (8.1) and (7.8) with $K(a)=c_{a}(\rho \sigma)^{-s(a)}$, where the constant $c_{a}$ and polynomial $s(a)$ may be increased a finite number of times.

From (3.6) and (7.2) we have

$$
\left\|I_{1}\right\|_{\tilde{\rho}(1), a}=\left\|\left(I-S_{t}\right) \bar{\partial}_{X} Z\right\|_{\tilde{\rho}(1), a} \leq c_{m} \rho^{-a} t^{b-a}\left\|\bar{\partial}_{X} Z\right\|_{\rho, b},
$$

for $0<t \leq \hat{c}^{-1} \rho \sigma, a \leq b$. We set $b=a+\mu \leq m$, then

$$
\left\|I_{1}\right\|_{\tilde{\rho}(1), a} \leq c_{m} \rho^{-a} t^{\mu}\left\|\bar{\partial}_{X} Z\right\|_{\rho, a+\mu},
$$

which, of course, is also valid for $a=k$.

For the commutator term $I_{3}=\left[S_{t}, \bar{\partial}_{M}\right] P \bar{\partial}_{X} Z$, lemma 7.1 gives

$$
\begin{aligned}
\left\|I_{3}\right\|_{\tilde{\rho}(2), k} & \leq c_{m} \rho^{-k-\alpha} t^{\alpha}\left\{\left\|P \bar{\partial}_{X} Z\right\|_{\tilde{\rho}(1), k+\alpha}+\|h\|_{\tilde{\rho}(1), k+2}\left\|P \bar{\partial}_{X} Z\right\|_{\tilde{\rho}(1), \alpha}\right\} \\
\left\|I_{3}\right\|_{\tilde{\rho}(2), a} & \leq c_{m} \rho^{-a}\left\{\left\|P \bar{\partial}_{X} Z\right\|_{\tilde{\rho}(1), a}+\|h\|_{\tilde{\rho}(1), a+2}\left\|P \bar{\partial}_{X} Z\right\|_{\tilde{\rho}(1), 0}\right\}
\end{aligned}
$$

In (9.3) we take $\alpha=1 / 2, \beta=5 / 2$ and use (7.9), (7.10),

$$
\begin{aligned}
\left\|P \bar{\partial}_{X} Z\right\|_{\tilde{\rho}(1), k+1 / 2} \leq & K(k)\left(1+\|h\|_{\rho, \beta}\right)\left(\left\|\bar{\partial}_{X} Z\right\|_{\rho, k}+\right. \\
& \left.+\|h\|_{\rho, k+\beta}\left\|\bar{\partial}_{X} Z\right\|_{\rho, 0}\right), \\
\left\|P \bar{\partial}_{X} Z\right\|_{\tilde{\rho}(1), 1 / 2} \leq & c_{1} \rho^{-3 / 2} \sigma^{-2 n}\left\|\bar{\partial}_{X} Z\right\|_{\rho, 0} .
\end{aligned}
$$


Thus,

$$
\left\|I_{3}\right\|_{\tilde{\rho}(2), k} \leq K(m)\left(1+\|h\|_{\rho, \beta}\right) t^{1 / 2}\left\{\left\|\bar{\partial}_{X} Z\right\|_{\rho, k}+\|h\|_{\rho, k+\beta}\left\|\bar{\partial}_{X} Z\right\|_{\rho, 0}\right\} .
$$

In (9.4) we use instead (7.8) to get

$$
\left\|I_{3}\right\|_{\tilde{\rho}(2), a} \leq K(a)\left\{\left\|\bar{\partial}_{X} Z\right\|_{\rho, a}+\|h\|_{\rho, a+2}\left\|\bar{\partial}_{X} Z\right\|_{\rho, 0}\right\}
$$

Next, $I_{2} \approx\left(X_{\bar{\alpha}}-Y_{\bar{\alpha}}\right) S_{t} P \bar{\partial}_{X} Z$, so lemma 6.1 gives

$$
\begin{aligned}
\left\|I_{2}\right\|_{\tilde{\rho}(2), a} \leq & c_{a} \rho^{-2 a}\left\{\left\|\bar{\partial}_{X} Z\right\|_{\tilde{\rho}(2), 0}\left\|S_{t} P \bar{\partial}_{X} Z\right\|_{\tilde{\rho}(2), a+1}+\right. \\
& \left.\left(\left\|\bar{\partial}_{X} Z\right\|_{\tilde{\rho}(2), 0}\|h\|_{\tilde{\rho}(2), a+1}+\left\|\bar{\partial}_{X} Z\right\|_{\tilde{\rho}(2), a}\right)\left\|S_{t} P \bar{\partial}_{X} Z\right\|_{\tilde{\rho}(2), 1}\right\} .
\end{aligned}
$$

First we take $a=k$ in (9.8), and use (7.2) with $a=k+1, b=k+1 / 2$, and (7.9),

$$
\begin{aligned}
\left\|S_{t} P \bar{\partial}_{X} Z\right\|_{\tilde{\rho}(2), k+1} \leq & c_{k} \rho^{-k-1} t^{-1 / 2}\left\|P \bar{\partial}_{X} Z\right\|_{\tilde{\rho}(1), k+1 / 2} \\
\leq & K(k)\left(1+\|h\|_{\rho, \beta}\right) t^{-1 / 2}\left(\left\|\bar{\partial}_{X} Z\right\|_{\rho, k}+\right. \\
& \left.+\|h\|_{\rho, k+\beta}\left\|\bar{\partial}_{X} Z\right\|_{\rho, 0}\right) \\
\left\|S_{t} P \bar{\partial}_{X} Z\right\|_{\tilde{\rho}(2), 1} \leq & K(1)\left(1+\|h\|_{\rho, \beta}\right)^{2} t^{-1 / 2}\left\|\bar{\partial}_{X} Z\right\|_{\rho, 0} .
\end{aligned}
$$

From this we get

$$
\begin{aligned}
\left\|I_{2}\right\|_{\tilde{\rho}(2), k} \leq & K(k)\left(1+\|h\|_{\rho, \beta}\right)^{2} t^{-1 / 2}\left\|\bar{\partial}_{X} Z\right\|_{\rho, 0}\left\{\left\|\bar{\partial}_{X} Z\right\|_{\rho, k}+\right. \\
& \left.+\|h\|_{\rho, k+\beta}\left\|\bar{\partial}_{X} Z\right\|_{\rho, 0}\right\} .
\end{aligned}
$$

With $a$ real in (9.8) we use $b=a<a+1$ in (7.2) and (7.8),

$$
\begin{aligned}
\left\|S_{t} P \bar{\partial}_{X} Z\right\|_{\tilde{\rho}(2), a+1} & \leq K(a) t^{-1}\left\{\left\|\bar{\partial}_{X} Z\right\|_{\rho, a}+\|h\|_{\rho, a+2}\left\|\bar{\partial}_{X} Z\right\|_{\rho, 0}\right\} \\
\left\|S_{t} P \bar{\partial}_{X} Z\right\|_{\tilde{\rho}(2), 1} & \leq K(0) t^{-1}\left\|\bar{\partial}_{X} Z\right\|_{\rho, 0} .
\end{aligned}
$$

This results in

$$
\left\|I_{2}\right\|_{\tilde{\rho}(2), a} \leq K(a) t^{-1}\left\|\bar{\partial}_{X} Z\right\|_{\rho, 0}\left\{\left\|\bar{\partial}_{X} Z\right\|_{\rho, a}+\|h\|_{\rho, a+2}\left\|\bar{\partial}_{X} Z\right\|_{\rho, 0}\right\}
$$

Finally we estimate $I_{4}=S_{t} Q \bar{\partial}_{M} \bar{\partial}_{X} Z$. With $a=k \geq 1, b=k-1 / 2$ in (7.2), and (7.9), we get

$$
\begin{aligned}
\left\|I_{4}\right\|_{\tilde{\rho}(2), k} \leq & c_{k} \rho^{-k} t^{b-k}\left\|Q \bar{\partial}_{M} \bar{\partial}_{X} Z\right\|_{\tilde{\rho}(1), b} \\
\leq & K(k)\left(1+\|h\|_{\rho, \beta}\right) t^{-1 / 2}\left\{\left\|\bar{\partial}_{M} \bar{\partial}_{X} Z\right\|_{\rho, k-1}+\right. \\
& \left.+\|h\|_{\rho, k-1+\beta}\left\|\bar{\partial}_{M} \bar{\partial}_{X} Z\right\|_{\rho, 0}\right\} .
\end{aligned}
$$

With $b=a-1$ in (7.2), and (7.8), we get

$$
\left\|I_{4}\right\|_{\tilde{\rho}(2), a} \leq K(a) t^{-1}\left\{\left\|\bar{\partial}_{M} \bar{\partial}_{X} Z\right\|_{\rho, a-1}+\|h\|_{\rho, a+1}\left\|\bar{\partial}_{M} \bar{\partial}_{X} Z\right\|_{\rho, 0}\right\}
$$


Using lemma 6.1, we get

$$
\begin{aligned}
\left\|\bar{\partial}_{M} \bar{\partial}_{X} Z\right\|_{\rho, a-1}= & \left\|\left(\bar{\partial}_{M}-\bar{\partial}_{X}\right) \bar{\partial}_{X} Z\right\|_{\rho, a-1} \\
\leq & c_{a} \rho^{-2(a-1)}\left\{\left\|\bar{\partial}_{X} Z\right\|_{\rho, 0}\left\|\bar{\partial}_{X} Z\right\|_{\rho, a}+\right. \\
& \left.+\left(\left\|\bar{\partial}_{X} Z\right\|_{\rho, 0}\|h\|_{\rho, a}+\left\|\bar{\partial}_{X} Z\right\|_{\rho, a-1}\right)\left\|\bar{\partial}_{X} Z\right\|_{\rho, 1}\right\} .
\end{aligned}
$$

We may use the convexity estimate (5.3) to absorb the last term into the first. We get

$$
\begin{aligned}
\left\|\bar{\partial}_{M} \bar{\partial}_{X} Z\right\|_{\rho, a-1} & \leq c_{a} \rho^{-3 a}\left\|\bar{\partial}_{X} Z\right\|_{\rho, 0}\left(\left\|\bar{\partial}_{X} Z\right\|_{\rho, a}+\|h\|_{\rho, a}\left\|\bar{\partial}_{X} Z\right\|_{\rho, 1}\right) \\
\left\|\bar{\partial}_{M} \bar{\partial}_{X} Z\right\|_{\rho, 0} & \leq c_{0}\left\|\bar{\partial}_{X} Z\right\|_{\rho, 0}\left\|\bar{\partial}_{X} Z\right\|_{\rho, 1} .
\end{aligned}
$$

This leads to

$$
\begin{aligned}
\left\|I_{4}\right\|_{\tilde{\rho}(2), k} \leq & K(k)\left(1+\|h\|_{\rho, \beta}\right) t^{-1 / 2}\left\|\bar{\partial}_{X} Z\right\|_{\rho, 0}\left\{\left\|\bar{\partial}_{X} Z\right\|_{\rho, k}+\right. \\
& \left.+\|h\|_{\rho, k-1+\beta}\left\|\bar{\partial}_{X} Z\right\|_{\rho, 1}\right\} \\
\left\|I_{4}\right\|_{\tilde{\rho}(2), a} \leq & K(a) t^{-1}\left\|\bar{\partial}_{X} Z\right\|_{\rho, 0}\left\{\left\|\bar{\partial}_{X} Z\right\|_{\rho, a}+\|h\|_{\rho, a+1}\left\|\bar{\partial}_{X} Z\right\|_{\rho, 1}\right\} .
\end{aligned}
$$

Combining (9.2) with $a=k,(9.6),(9.10)$, and (9.18), and simplifying slightly gives the following fine four-term estimate.

Lemma 9.1. With $k \in \mathbf{Z}, 1 \leq k, k+\mu \leq m, \beta=5 / 2$, we have

$$
\begin{aligned}
\left\|\bar{\partial}_{X}(Z+F)\right\|_{\tilde{\rho}(2), k} \leq & K(m)\left(1+\|h\|_{\rho, \beta}\right)^{2}\left\{t^{\mu}\left\|\bar{\partial}_{X} Z\right\|_{\rho, k+\mu}+\right. \\
& +t^{1 / 2}\left(\left\|\bar{\partial}_{X} Z\right\|_{\rho, k}+\|h\|_{\rho, k+\beta}\left\|\bar{\partial}_{X} Z\right\|_{\rho, 0}\right)+ \\
& \left.+t^{-1 / 2}\left\|\bar{\partial}_{X} Z\right\|_{\rho, 0}\left(\left\|\bar{\partial}_{X} Z\right\|_{\rho, k}+\|h\|_{\rho, k+\beta}\left\|\bar{\partial}_{X} Z\right\|_{\rho, 1}\right)\right\}
\end{aligned}
$$

Combining (9.2) with $\mu=0,(9.7),(9.13)$, and (9.19) gives the following coarse four-term estimate.

Lemma 9.2. For $1 \leq a \leq m$,

$$
\begin{aligned}
\left\|\bar{\partial}_{X}(Z+F)\right\|_{\tilde{\rho}(2), a} \leq & K(m)\left(1+t^{-1}\left\|\bar{\partial}_{X} Z\right\|_{\rho, 0}\right) \cdot \\
& \cdot\left\{\left\|\bar{\partial}_{X} Z\right\|_{\rho, a}+\|h\|_{\rho, a+2}\left\|\bar{\partial}_{X} Z\right\|_{\rho, 1}\right\} .
\end{aligned}
$$

In the general estimate of lemma 6.2 , we take $\rho_{1}=\rho(1-5 \sigma)$ and the domain $D_{\rho_{1}}\left(h_{1}\right)$ on the left hand side. The first term on the right is estimated by (9.20) for the following, which constitutes the fine estimate for $\bar{\partial}_{X^{1}} Z_{1}$.

\section{Lemma 9.3.}

$$
\begin{aligned}
\left\|\bar{\partial}_{X^{1}} Z_{1}\right\|_{\rho_{1}, k} \leq & K(m)\left(1+\|h\|_{\rho, \beta}\right)^{2}\left\{t^{\mu}\left\|\bar{\partial}_{X} Z\right\|_{\rho, k+\mu}+\right. \\
& +\left(t^{1 / 2}+t^{-1 / 2}\left\|\bar{\partial}_{X} Z\right\|_{\rho, 1}+t^{-1}\left\|\bar{\partial}_{X} Z\right\|_{\rho, 0}\right) . \\
& \left.\cdot\left(\left\|\bar{\partial}_{X} Z\right\|_{\rho, k}+\|h\|_{\rho, k+\beta}\left\|\bar{\partial}_{X} Z\right\|_{\rho, 0}\right)\right\} .
\end{aligned}
$$


Proof. We must estimate the remaining terms in (6.3) with $a=k$. We use (8.4) with $a=b=k$ for $\|F\|_{\tilde{\rho}(2), k}$, and (8.3) for $\|F\|_{\tilde{\rho}(2), 1}$. This gives

$$
\begin{aligned}
\|F\|_{\tilde{\rho}(2), k}+\|F\|_{\tilde{\rho}(2), 1}\|h\|_{\rho, k} \leq & K(k)\left\{\left\|\bar{\partial}_{X} Z\right\|_{\rho, k}+\right. \\
& \left.+2 t^{-1 / 2}\left\|\bar{\partial}_{X} Z\right\|_{\rho, 0}\|h\|_{\rho, k+2}\right\}, \\
\left\|\bar{\partial}_{X} Z\right\|_{\rho, 0}+\|F\|_{\tilde{\rho}(2), 1} \leq & 2 K(1) t^{-1 / 2}\left\|\bar{\partial}_{X} Z\right\|_{\rho, 0} .
\end{aligned}
$$

For $\|F\|_{\tilde{\rho}(2), k+1}$ we use (8.5) with $a=k+1, l=k$, to get

$$
\begin{aligned}
\|F\|_{\tilde{\rho}(2), k+1}+\|F\|_{\tilde{\rho}(2), 1}\|h\|_{\rho, k+1} \leq & K(k+1)\left(1+\|h\|_{\rho, \beta}\right) t^{-1 / 2} . \\
& \left.\cdot\left(\left\|\bar{\partial}_{X} Z\right\|_{\rho, k}++\|h\|_{\rho, k+\beta}\left\|\bar{\partial}_{X} Z\right\|_{\rho, 0}\right)\right\} .
\end{aligned}
$$

Combining gives the lemma.

For a coarse estimate of the error, we combine lemmas 8.2 and 9.2 , and simplify.

Lemma 9.4. For $1 \leq a \leq m$,

$$
\begin{aligned}
\left\|\bar{\partial}_{X^{1}} Z_{1}\right\|_{\rho_{1}, a} \leq & K(m+1)\left(1+\left(1+\|h\|_{\rho, 3}\right)^{2} t^{-1}\left\|\bar{\partial}_{X} Z\right\|_{\rho, 1}\right) . \\
& \cdot\left(\left\|\bar{\partial}_{X} Z\right\|_{\rho, a}+\|h\|_{\rho, a+2}\left\|\bar{\partial}_{X} Z\right\|_{\rho, 1}\right) .
\end{aligned}
$$

\section{The sequence of embeddings. Summary.}

Now we begin the process of inductively constructing a sequence of approximately holomorphic embeddings and showing convergence. For $j \geq 0$ we set, according to the second paragraph of section 8,

$$
\rho_{j+1}=\rho_{j}\left(1-5 \sigma_{j}\right), \quad \tilde{\rho}_{j}(l)=\rho_{j}\left(1-\sigma_{j}\right)^{l}, \quad \sigma_{j+1}=5^{-1} \sigma_{j},
$$

with $\sigma_{0}=5^{-2}, 0<l \leq 4$, and $\rho_{0}>0$ free to be determined. We choose $\rho_{0} \leq 1$ so that $D_{\rho_{0}} \subset D_{1}^{0}$, where $D_{1}^{0}$ is the domain of lemma 1.2. Clearly the $\rho_{j}$ decrease to a positive limit $\rho_{\infty}>0$. We also set

$$
K_{j}(a)=c_{a}\left(\rho_{j} \sigma_{j}\right)^{-s(a)} ; \quad N_{j}(a)=1+\left\|h_{j}\right\|_{\rho_{j}, a} ; \quad \delta_{j}(a)=\left\|\bar{\partial}_{X^{j}} Z_{j}\right\|_{\rho_{j}, a},
$$

where we must take $a \leq m$ in the last. We note that $h_{0}=0$, and $\delta_{0}(m)$ can be made arbitrarily small by non-isotropic dilation. We readily see that

$$
K_{j+1}(a) \leq \hat{c}_{a} K_{j}(a)
$$

We shall choose the smoothing parameters $t_{j}$ by

$$
t_{j+1}=t_{j}^{\kappa}, \quad 1<\kappa, \quad 0<t_{0}<1
$$


with $t_{0}$ sufficiently small. Then the $t_{j}$ strictly decrease rapidly to zero. Notice that $\hat{c} t_{j+1} / \rho_{j+1} \sigma_{j+1} \leq\left(\hat{c} t_{j} / \rho_{j} \sigma_{j}\right) t_{0}^{\kappa-1}\left(5 / 1-5 \sigma_{0}\right)$, so that

$$
0<t_{j}<\hat{c}^{-1} \rho_{j} \sigma_{j},(\hat{c}=5 / \sqrt{2}),
$$

for all $j$, if $t_{0}$ is sufficiently small, $\rho_{0}=1$ being fixed. (We shall have to shrink $t_{0}$ a finite number of times.)

We want to construct $h_{j}, Z_{j} \in C^{\infty}\left(D_{\rho_{j}}\right)$, and $X_{\alpha}^{j} \in C^{m}\left(D_{\rho_{j}}\right)$, as in (1.4), (1.5) and (2.2). To pass from $j$ to $j+1$, we need to make the inductive assumption

$$
\left\|h_{j}\right\|_{\rho_{j}, 2} \leq \gamma_{0}
$$

in order to apply lemma 2.1 and to make use of the results of section 7 . Then we may use the solution operator $P=P_{j}$ and smoothing operator $S_{t_{j}}$ to construct $F_{j}=-S_{t_{j}} P_{j} \bar{\partial}_{X^{j}} Z_{j}$.

To construct our sequences, we shall choose a suitable integer $k, 1 \leq k \leq m$, and a real number $a, 2 \leq a, k \leq a$. The goal is to get convergence of $h_{j}, Z_{j}$ in the Hölder class $C^{a}$, while the error $\delta_{j}(k)$ goes to zero rapidly. We make the second inductive assumption,

$$
t_{j}^{-s} \delta_{j}(k) \leq 1
$$

for a suitable choice of $s>1 / 2$. Given this, we claim that the conditions

$$
t_{j}^{-1 / 2} \delta_{j}(0) \leq \gamma_{1} \rho_{j}^{7 / 2} \sigma_{j}^{2 n+1}, \quad \delta_{j}(1) \leq 1,
$$

will hold for all $j$, if $t_{0}>0$ is sufficiently small. The second is clear. To see the first, put $Q_{j}=t_{j}^{s-1 / 2} \rho_{j}^{-7 / 2} \sigma_{j}^{-2 n-1}$. By (10.7) we need $Q_{j} \leq \gamma_{1}$ for all $j$. Since $\kappa>1$, $s>1 / 2$ and the $t_{j}, \sigma_{j}$ decrease, we easily see that $Q_{0} \leq \gamma_{1}$ and $Q_{j+1} / Q_{j} \leq 1$, if $t_{0}$ is sufficiently small.

We start the process at $j=0$, using lemma 1.2 , so that $h_{0}=0$. Once $s, \kappa$ and $t_{0}>0$ have been fixed, we may apply non-isotropic dilation to make $\delta_{0}(m)$ as small as is needed. This will give (10.6), (10.7) for $j=0$. We then construct our sequences $h_{j}, Z_{j}$ of approximate CR embeddings, and verify (10.6), (10.7), inductively.

From (8.6), (8.7), lemma 8.1, lemma 9.3, and lemma 9.4, respectively, we have the following. They summarize the main estimates that have been derived up to this point. In them $\beta=5 / 2, b \leq a, b \leq m \in \mathbf{R}, k, l$ are integers $1 \leq k, l \leq m$, $k+\mu \leq m, l+(1 / 2) \leq a$.

$$
\begin{aligned}
\left\|f_{j(2)}\right\|_{\tilde{\rho}_{j}(2), a} \leq & K_{j}(a)\left\{t_{j}^{-1 / 2} \delta_{j}(0) N_{j}(a)+\right. \\
& \left.+t_{j}^{b-a}\left(\delta_{j}(b)+N_{j}(b+2) \delta_{j}(0)\right)\right\} \\
\left\|g_{j(2)}\right\|_{\rho_{j+1}, a} \leq & c_{a} \rho_{j}^{-4(a+2)}\left\|f_{j(2)}\right\|_{\tilde{\rho}_{j}(2), a}
\end{aligned}
$$


(10.11)

$$
\left\|h_{j+1}-h_{j}\right\|_{\rho_{j+1}, a} \leq K_{j}(a)\left\{t_{j}^{-1 / 2} \delta_{j}(0) N_{j}(a+1)+t_{j}^{b-a}\left(\delta_{j}(b)+N_{j}(b+2) \delta_{j}(0)\right)\right\}
$$

$(10.12) N_{j+1}(a) \leq K_{j}(a)\left\{N_{j}(a)+t_{j}^{b-a}\left(\delta_{j}(b)+N_{j}(b+2) \delta_{j}(0)\right)\right\}$,

$(10.13) N_{j+1}(a) \leq K_{j}(a)\left\{N_{j}(a)+t_{j}^{l+(1 / 2)-a}\left(N_{j}(\beta) \delta_{j}(l)+N_{j}(l+\beta) \delta_{j}(0)\right)\right\}$,

$$
\begin{aligned}
\delta_{j+1}(k) \leq & K_{j}(m) N_{j}(\beta)^{2}\left\{t_{j}^{\mu} \delta_{j}(k+\mu)+\right. \\
& \left.+\left(t_{j}^{1 / 2}+t_{j}^{-1 / 2} \delta_{j}(1)+t_{j}^{-1} \delta_{j}(0)\right) \delta_{j}(k) N_{j}(k+\beta)\right\},
\end{aligned}
$$

$$
\begin{aligned}
\delta_{j+1}(k+\mu) \leq & K_{j}(m+1)\left(1+N_{j}(3)^{2} t_{j}^{-1} \delta_{j}(1)\right) \\
& \cdot\left\{\delta_{j}(k+\mu)+N_{j}(k+\mu+2) \delta_{j}(1)\right\} \\
\delta_{j+1}(m) \leq & K_{j}(m+1)\left(1+N_{j}(3)^{2} t_{j}^{-1} \delta_{j}(1)\right) \\
& \cdot\left\{\delta_{j}(m)+N_{j}(m+2) \delta_{j}(1)\right\}
\end{aligned}
$$

\section{The main inductive hypotheses.}

In this section we verify inductively (10.6) and (10.7) for all $j$. For this we need to control the possible growth of the following norms.

Lemma 11.1. If $t_{j}^{-s} \delta_{j}(k) \leq 1$, with $s \geq 2$, then

$$
N_{j+1}(a) \leq 3 K_{j}(a) N_{j}(a)
$$

for $a=3, a=\beta, a=k+2, a=k+\beta$.

Proof. For the case $a=3$, we use (10.12) with $a=3$ and $b=1$, and (10.7). This gives $N_{j+1}(3) \leq K_{j}(3) N_{j}(3)\left\{1+2 t_{j}^{s-2}\right\}$, hence the estimate. For $a=k+2$, we use (10.12) with $b=k$. For $a=\beta$ or $a=k+\beta$, we use (10.13) with $l=0$, or $l=k$.

Now we assume that (10.6), (10.7) hold for all $j \leq l$ and verify (10.6) for $j=l+1$.

Lemma 11.2. Assume that (10.6) and (10.7) with $s \geq 2$ hold for all $j \leq l$. Then $\left\|h_{l+1}\right\|_{\rho_{l+1}, 2} \leq \gamma_{0}$, independently of $l$, if $t_{0}>0$ is chosen sufficiently small.

Proof. For the proof we apply (10.11) with $a=2, b=1$, and use (10.7). This gives

$$
\left\|h_{j+1}-h_{j}\right\|_{\rho_{j+1}, 2} \leq 3 K_{j}(2) N_{j}(3) t_{j}^{s-1} \equiv P_{j}
$$


Since $h_{0}=0$, it suffices to show $\sum_{j=0}^{l} P_{j} \leq \gamma_{0}$. From (11.1) with $a=3$, it follows that

$$
\begin{aligned}
P_{j+1} / P_{j} & \leq 3 \hat{c}_{2} K_{j}(3) t_{j}^{(\kappa-1)(s-1)} \equiv Q_{j}, \\
Q_{j+1} / Q_{j} & \leq \hat{c}_{3} t_{j}^{(\kappa-1)^{2}(s-1)} .
\end{aligned}
$$

Since $\rho_{0}, \sigma_{0}$ are fixed, this we see that $Q_{0}<1$, and $Q_{j+1} \leq \epsilon Q_{j}$, say $\epsilon=1 / 2$, if $t_{0}>0$ is chosen sufficiently small, depending on $\kappa, s$. Then $Q_{j} \leq \epsilon^{j}$, and for $j \geq 1$, $P_{j} \leq Q_{j-1} P_{j-1} \leq \cdots \leq \sqrt{\epsilon}^{j(j-1)} P_{0}$. Thus

$$
\sum_{j=0}^{l} P_{j} \leq P_{0}+\left(\sum_{j=1}^{\infty} \sqrt{\epsilon}^{j(j-1)}\right) P_{0} \leq P_{0} /(1-\sqrt{\epsilon}) .
$$

This will be less than or equal $\gamma_{0}$, if $t_{0}$ is sufficiently small, since $h_{0}=0$ and $N_{0}(3)=1$.

It remains to achieve the main inductive assumption (10.7) for all $j$. Thus we assume (10.7) holds for $j$ and verify it for $j+1$. Since $s \geq 2, t_{j}^{1 / 2}$ is largest of three terms, so (10.14) and (10.7) give

$$
\begin{aligned}
t_{j+1}^{-s} \delta_{j+1}(k) & \leq a_{j}+B_{j}, \\
a_{j} & =3 K_{j}(m) N_{j}(\beta)^{2} N_{j}(k+\beta) t_{j}^{(1-\kappa) s+1 / 2}, \\
B_{j} & =K_{j}(m) N_{j}(\beta)^{2} t_{j}^{\mu-\kappa s} \delta_{j}(k+\mu) .
\end{aligned}
$$

We want the two exponents of $t_{j}$ to be positive. Thus we shall choose $\mu>\kappa s$, $\alpha=(1-\kappa) s+1 / 2>0$, and $s=2$. This gives the restriction $1<\kappa<5 / 4$. Thus we choose

$$
s=2, \quad 1<\kappa<5 / 4, \quad \mu>\kappa s .
$$

The first step is to make $a_{j}<1 / 2$. Using lemma 11.1 as in the proof of lemma 11.2 , we get

$$
\begin{aligned}
& a_{j+1} / a_{j} \leq 27 \hat{c}_{m} K_{j}(\beta)^{2} K_{j}(k+\beta) t_{j}^{(\kappa-1) \alpha} \equiv \tilde{a}_{j}, \\
& \tilde{a}_{j+1} / \tilde{a}_{j} \leq \hat{c}_{\beta}^{2} \hat{c}_{k+\beta} t_{j}^{(\kappa-1)^{2} \alpha} .
\end{aligned}
$$

Thus, if $t_{0}$ is sufficiently small, all $\tilde{a}_{j}<1$, and all $a_{j}<1 / 2$. We shall use this kind of argument several more times. 
The second step is to make $B_{j}<1 / 2$ for all $j$. By (10.14), (10.7) and (10.16), and since $1+N_{j}(3)^{2} t_{j}^{s-1} \leq 2$, if $t_{0}$ is sufficiently small, we have

$$
\begin{aligned}
B_{j+1} & \leq b_{j} B_{j}+E_{j}, \\
b_{j} & =C_{j} t_{j}^{(\kappa-1)(\mu-\kappa s)}, \\
E_{j} & =C_{j} N_{j}(\beta)^{2} N_{j}(k+\mu+2) t_{j}^{\kappa(\mu-\kappa s)+s},
\end{aligned}
$$

where, for a common constant, we may take $C_{j}=18 \hat{c}_{m} K_{j}(\beta)^{2} K_{j}(m+1)^{2}$. Since $\mu>\kappa s$, both of the exponents are positive. Since $C_{j+1} / C_{j}=\hat{C}_{0}$, we see that $b_{j} \leq 1 / 2$ for all $j$, if $t_{0}$ is sufficiently small.

If we can show that $E_{j} \leq 1 / 4$ for all $j$, then $B_{j}<1 / 2$ for all $j$. To check its possible growth, we use (10.13) with $a=k+\mu+2$ and $l=k$. With $\gamma=\kappa(\mu-\kappa s)+s$, we get

$$
\begin{aligned}
E_{j+1} & \leq e_{j} E_{j}+f_{j}, \\
e_{j} & =C_{j}^{\prime} t_{j}^{(\kappa-1) \gamma}, \\
f_{j} & =C_{j}^{\prime \prime} N_{j}(3)^{2} N_{j}(k+\beta) t_{j}^{\kappa \gamma+s-\mu-3 / 2},
\end{aligned}
$$

where $C_{j}^{\prime}=\hat{C}_{0}\left(3 K_{j}(\beta)\right)^{2} K_{j}(k+\mu+2)$ and $C_{j}^{\prime \prime}=C_{j}^{\prime} C_{j}$. Clearly we can arrange $e_{j}<1 / 2$ by choosing $t_{0}$ sufficiently small. By lemma 11.1 we can make $f_{j}<1 / 8$ for all $j$, provided the exponent of $t_{j}$ is positive. But a simple computation shows that $\kappa \gamma+s-\mu-3 / 2>0$, given (11.9).

Hence, if $t_{0}>0$ is sufficiently small, then $B_{j}<1 / 2$ for all $j$, if (11.19) holds. This proves the following.

Lemma 11.3. Suppose that $s=2,1<\kappa<5 / 4$ and $\mu>\kappa s$. Let $1 \leq k \leq m, 2 \leq a$, $k \leq a$. If $t_{0}>0$ and then $\delta_{0}(k)$ are taken sufficiently small, (10.6) and (10.7) will hold for all $j$.

Explicitly, we require $\mu>\kappa s, 5 / 2>\kappa s>2$, and $k+\mu \leq m$.

\section{Convergence in $C^{a}$.}

Now we assume that $k \leq a$ and the other parameters are chosen as in the last lemma. We want to show that our sequence of approximate CR-embeddings, $h_{j}$, $Z_{j}$ on $D_{\rho_{j}}\left(h_{j}\right)$, converges in $C^{a}$-norm on some neighborhood of 0. By lemma 2.1 all these domains contain the ball of radius $\sqrt{2 / 3} \rho_{\infty}$. By (10.11) with $b=k$, it suffices to show that

$$
\sum_{j=1}^{\infty} K_{j}(a) \delta_{j}(k)\left\{t_{j}^{-1 / 2} N_{j}(a+1)+2 t_{j}^{k-a} N_{j}(k+2)\right\}<\infty
$$


Using (10.7) it suffices to show both

$$
\sum_{j=1}^{\infty} K_{j}(a) N_{j}(a+1) t_{j}^{s-1 / 2}<\infty, \quad \sum_{j=1}^{\infty} K_{j}(a) N_{j}(k+2) t_{j}^{k-a+s}<\infty .
$$

We apply the ratio test to the second, then first series.

$$
\begin{aligned}
\frac{K_{j+1}(a) N_{j+1}(k+2) t_{j+1}^{k-a+s}}{K_{j}(a) N_{j}(k+2) t_{j}^{k-a+s}} \leq & 2 \hat{c}_{j}(a) K_{j}(k+2) t_{j}^{(\kappa-1)(s+k-a)}, \\
\frac{K_{j+1}(a) N_{j+1}(a+1) t_{j+1}^{s-1 / 2}}{K_{j}(a) N_{j}(a+1) t_{j}^{s-1 / 2}} \leq & \hat{c}_{j}(a) K_{j}(a+1) t_{j}^{(\kappa-1)(s-1 / 2)}\{1+ \\
& \left.+t_{j}^{k-a-1+s} N_{j}(k+2) / N_{j}(a+1)\right\} .
\end{aligned}
$$

With $a \geq k+1$, the right-hand sides will go to zero, if the exponents of $t_{j}$ are positive. Thus we need $k+s>a$ and $k+s-1+(\kappa-1)(s-1 / 2)>a$, that is $k+2>a$ and $k+1+(3 / 2)(\kappa-1)>a$. In particular, we can take $a=k+1$.

The restriction on the integer $m$ comes from $m \geq k+\mu, k \geq 1, \mu>\kappa s$, $5 / 2>\kappa s>2$. Thus we need $m>3$ to run the argument. We put $m=3+\eta, \eta>0$. Then we need

$$
\begin{aligned}
& 1<\kappa<\min (5 / 4,(\eta+3-k) / 2) \leq \min (5 / 4,1+(\eta / 2)) \\
& 2<\kappa s<\mu<m-k
\end{aligned}
$$

For $m>3$, we fix such a $\kappa$, and $\mu$, and run the argument.

We summarize what has been achieved thus far. We have constructed a sequence of $C^{\infty}$-smooth real hypersurfaces $M_{j}: y^{n}=\left|z^{\prime}\right|^{2}+h_{j}\left(z^{\prime}, x^{n}\right)$, and embeddings $Z_{j}=\left(z^{\prime}, x^{n}+i\left(\left|z^{\prime}\right|^{2}+h_{j}\left(z^{\prime}, x^{n}\right)\right)\right.$ of a neighborhood $D_{\infty}$ of 0 in the real hyperplane $\operatorname{Im}\left(z^{n}\right)=0$, into $\mathbf{C}^{n}$. Our original CR structure, or complex vector frame field $X_{\alpha}$ of class $C^{m}$ has also been transplanted to $D_{\infty}$, where it is subjected to a sequence of diffeomorphisms and frame changes to get the sequence $X_{\alpha}^{j}$ of complex vector frame fields. We have $X_{\alpha}^{j}=Y_{\alpha}^{j}+A_{\alpha}^{\bar{\beta}} Y_{\bar{\beta}}^{j}+B_{\alpha} \partial_{x^{n}}$, as in (1.8), (1.9), where the $Y_{\bar{\beta}}$, given by (1.7), are the tangential CR operators to $M_{j}$.

We have shown that $h_{j} \rightarrow h_{\infty}$ and $Z_{j} \rightarrow Z_{\infty}$ in $C^{a}\left(D_{\infty}\right)$ norm, while $X_{\bar{\alpha}}^{j} Z_{j} \rightarrow 0$, rapidly. It follows that $Y_{\alpha}^{j} \rightarrow Y_{\alpha}^{\infty}$ in $C^{a-1}=C^{k}$ norm. By $(1.9)\left(A_{\alpha}^{\bar{\beta}}, B_{\alpha}\right) \rightarrow 0$ (rapidly). Hence, $X_{\alpha}^{j} \rightarrow X_{\alpha}^{\infty} \equiv Y_{\alpha}^{\infty}$, in $C^{k}$ norm. These limiting vector fields are of class $C^{a-1}=C^{k}$.

We claim that the complex vector frame field $X_{\alpha}^{\infty} \equiv Y_{\alpha}^{\infty}$ spans a CR structure equivalent to our original CR structure. The equivalence will be given by the composition $\tilde{f}_{\infty}$ of all the maps $f_{j}$. This will be shown to converge in the appropriate norms in the final section. Then $Z_{\infty} \circ \tilde{f}_{\infty}$ will be the required CR embedding. This argument will yield the following. 
Proposition 12.1. Let $X_{\bar{\alpha}}, 1 \leq \alpha \leq n-1$, be local complex vector fields on $\mathbf{R}^{2 n-1}, 2 n-1 \geq 7$, which represent a formally integrable, strongly pseudoconvex $C R$ structure of class $C^{m}, m \in \mathbf{R}, 3<m \leq \infty$. Then there exists a local $C R$ embedding. It is of class $C^{a}$, for all $a, 0 \leq a<m$.

The arguments of this and the final section give the result with $0 \leq a<m-\mu+1$. The next section improves the regularity of the solutions to that stated in the proposition and theorem 0.1 .

\section{Interpolation and higher regularity.}

Now let $m_{0} \in \mathbf{R}, m_{0}>3,2 \leq a=k+1 \leq m_{0}-\mu+1$, and $X_{\alpha}$ of class $C^{m_{0}}$, and $h_{j}, Z_{j}$ converging in $C^{a}\left(D_{\infty}\right)$ as in proposition 12.1 . Let $m \in \mathbf{Z}$ with $m \geq m_{0}$.

We want to investigate the convergence of these same sequences also in $C^{b}\left(D_{\infty}\right)$, for

$$
a<b<m+(1 / 2), \quad b=\lambda a+(1-\lambda)(m+(1 / 2)), \quad 0<\lambda<1,
$$

for any $m \geq m_{0}$ for which the original $X_{\alpha}$ are of class $C^{m}$. We shall apply the ratio test to the series $\sum\left\|h_{j+1}-h_{j}\right\|_{b}$, where the norms are over the domain $D_{\infty}$. For this we use the interpolation inequality (5.2) to get

$$
\begin{aligned}
\left\|h_{j+1}-h_{j}\right\|_{b} & \leq c_{m} \rho_{j}^{-m} A_{j}^{\lambda} B_{j}^{(1-\lambda)}, \\
A_{j} & =\left\|h_{j+1}-h_{j}\right\|_{\rho_{j+1}, a}, \\
B_{j} & =\left\|h_{j+1}-h_{j}\right\|_{\rho_{j+1}, m+(1 / 2)} \\
& \leq N_{j+1}(m+(1 / 2))+N_{j}(m+(1 / 2)) .
\end{aligned}
$$

We have $\rho_{j} \geq \rho_{\infty}>0$, and we know that $A_{j}$ goes to zero rapidly. In fact,as in (12.2), taking $a=k+1$ and $b=k$ in (10.11) and using (10.7) and lemma 11.1 gives (increasing $A_{j}$ )

$$
\begin{aligned}
A_{j} & =2 K_{j}(k+1) N_{j}(k+2) t_{j}^{s-1}, \\
A_{j+1} / A_{j} & \leq 3 \hat{c}_{k+1} K_{j}(k+2) t_{j}^{(\kappa-1)(s-1)} .
\end{aligned}
$$

We must control the possible growth of $B_{j}$.

Taking $a=m+(1 / 2)$, then $a=m+\beta$, and $l=m$ in (10.13) and using (10.7) gives

$$
\begin{aligned}
N_{j+1}(m+(1 / 2)) \leq & K_{j}(m+(1 / 2))\left\{N_{j}(m+(1 / 2))+\right. \\
& \left.+N_{j}(\beta) \delta_{j}(m)+t_{j}^{s} N_{j}(m+\beta)\right\} . \\
N_{j+1}(m+\beta) \leq & K_{j}(m+\beta)\left\{N_{j}(m+\beta)+\right. \\
& \left.+t_{j}^{-2}\left[N_{j}(\beta) \delta_{j}(m)+t_{j}^{s} N_{j}(m+\beta)\right]\right\} .
\end{aligned}
$$


By increasing $B_{j}$, we may write

$$
\begin{aligned}
B_{j} & =E_{j}+F_{j}+G_{j}, \\
E_{j} & =2 K_{j}(m+(1 / 2)) N_{j}(m+(1 / 2)), \\
F_{j} & =K_{j}(m+(1 / 2)) N_{j}(\beta) \delta_{j}(m), \\
G_{j} & =K_{j}(m+(1 / 2)) N_{j}(m+\beta) t_{j}^{s} .
\end{aligned}
$$

We next derive the following growth estimates. The first follows directly from (13.7) and the definitions.

$$
\begin{aligned}
E_{j+1} & \leq 2 \hat{c}_{m+(1 / 2)} K_{j}(m+(1 / 2)) B_{j} \\
F_{j+1} & \leq 12 \hat{c}_{m+(1 / 2)} K_{j}(\beta) K_{j}(m+1) N_{j}(\beta) B_{j} . \\
G_{j+1} & \leq 2 \hat{c}_{m+(1 / 2)} K_{j}(m+\beta) t_{j}^{2(\kappa-1)} B_{j} .
\end{aligned}
$$

For the third we use (13.8), $s=2$, and $\kappa s-2 \geq 0$. For the second we use (10.16), (10.7), and $1+N_{j}(3)^{2} t_{j}^{s-1} \leq 2$, as arranged in section 11 . We get

$$
\delta_{j+1}(m) \leq 2 K_{j}(m+1)\left\{\delta_{j}(m)+N_{j}(m+2) t_{j}^{s}\right\} .
$$

From this we get

$$
F_{j+1} \leq 6 \hat{c}_{m+(1 / 2)} K_{j}(\beta) K_{j}(m+1)\left(F_{j}+N_{j}(\beta) G_{j}\right)
$$

which gives (13.14). Combining gives

$$
B_{j+1} \leq 16 \hat{c}_{m+(1 / 2)} K_{j}(m+\beta) K_{j}(\beta) N_{j}(\beta) B_{j} .
$$

From (13.6) and (13.18) we see that

$$
\left(A_{j+1} / A_{j}\right)^{\lambda}\left(B_{j+1} / B_{j}\right)^{1-\lambda} \leq \tilde{C}_{j} t_{j}^{\alpha \lambda}
$$

where $\alpha>0$,and $\tilde{C}_{j}$ is slowly growing. Since $0<\lambda$, (13.19) tends to zero, as $j \rightarrow \infty$. Hence, we have convergence in $C^{b}\left(D_{\infty}\right)$.

Thus, if our original vector fields $X_{\alpha}$ are of class $C^{m}, \infty>m \geq m_{0}>3$, then the limiting real hypersurface $M_{\infty}: \quad y^{n}=\left|z^{\prime}\right|^{2}+h_{\infty}\left(z^{\prime}, x^{n}\right)$, and CR embedding $Z_{\infty}=\left(z^{\prime}, x^{n}+i\left(\left|z^{\prime}\right|^{2}+h_{\infty}\left(z^{\prime}, x^{n}\right)\right)\right)$, are of class $C^{b}$ for all $b<m+1 / 2$. In the $C^{\infty}$ case, we may apply the same argument, with perhaps different constants appearing in the estimates, for each $m>m_{0}$, to the fixed sequence. Thus, $M_{\infty}$ and $Z_{\infty}$ are class $C^{\infty}$. Combined with proposition 12.1, this gives theorem 0.1 .

In case $m \in \mathbf{R}, m>3$, is not an integer, an entirely similar but simpler argument, using the estimate for $N_{j}(m)$ gotten from (10.12) with $a=b=m$ gives that the embedding of proposition 12.1 is of class $C^{a}, 0 \leq a<m$. 


\section{Composition of mappings.}

In this section we show that the sequence of compositions of the maps $f_{j}$ actually converges on some neighborhood of 0 in $\mathbf{R}^{2 n-1}$. The limiting map will provide a CR-equivalence of our original structure $X_{\alpha}$ and the embedded structure $X_{\alpha}^{\infty}$ of section 12. With it we shall get the solutions $z^{j}$ of $(0.1)$ for the original vector fields $X_{\bar{\alpha}}$. The argument can be motivated by a similar but much simpler one given in $[22]$.

We consider the sequences of compositions of mappings,

$$
\begin{gathered}
\tilde{f}_{j}=f_{j} \circ f_{j-1} \circ \cdots \circ f_{0}: \tilde{U}_{j} \rightarrow \mathbf{R}^{2 n-1}, \\
\tilde{g}_{j}=g_{0} \circ g_{1} \circ \cdots \circ g_{j}: D_{\rho_{j+1}} \rightarrow D_{\rho_{0}},
\end{gathered}
$$

where $\tilde{U}_{j}=\tilde{g}_{j}\left(D_{\rho_{j+1}}\right)$. The domains $\tilde{U}_{j} \subseteq U_{j} \subset D_{\tilde{\rho}_{j}(2)}$ (see lemma 4.1 and (8.1)) are decreasing, compact, smoothly bounded neighborhoods of 0 . The domains $D_{\rho_{j}}$ are decreasing, compact, strictly convex, and $D_{\rho_{j}} \supseteq B\left(\sqrt{2 / 3} \rho_{j}\right)$, and $D_{\infty}=\cap D_{\rho_{j}} \supseteq$ $B\left(\sqrt{2 / 3} \rho_{\infty}\right)$ is a compact convex neighborhood of 0 .

To investigate the convergence of the sequences $\tilde{f}_{j}, \tilde{g}_{j}$, we let $d$ denote Jacobian matrix, and $|\cdot|_{U}$ denote the sup of the matrix operator norm over the set $U$. Then, since $\tilde{g}_{j}=\tilde{g}_{j-1} \circ g_{j}$,

$$
\begin{aligned}
d \tilde{g}_{j} & =\left(d \tilde{g}_{j-1} \circ g_{j}\right) d g_{j} \\
\left|d \tilde{g}_{j}\right|_{\rho_{j+1}} & \leq\left|d \tilde{g}_{j-1}\right|_{\rho_{j}}\left|d g_{j}\right|_{\rho_{j+1}} \leq \cdots \leq \prod_{i=0}^{j}\left(1+\left|d g_{i(2)}\right|_{\rho_{i+1}}\right)
\end{aligned}
$$

The infinite product will converge iff $\sum_{j=0}^{\infty}\left|d g_{j(2)}\right|_{\rho_{j+1}}<\infty$, which will follow from $\sum_{j=0}^{\infty}\left\|g_{j(2)}\right\|_{\rho_{j+1}, k}<\infty$, since $k \geq 1$. But this follows from (10.10), and (10.9) with $a=b=k$, and (10.7), which give

$$
\left\|g_{j(2)}\right\|_{\rho_{j+1}, k} \leq K_{j}(k) t_{j}^{s-1 / 2} N_{j}(k+2),
$$

and lemma 11.1, and the ratio test. In particular, we have a uniform bound, $\left|d \tilde{g}_{j}\right|_{\rho_{j+1}} \leq C_{1}$ for all $j$.

As in the proof of lemma 4.2,

$$
\begin{aligned}
\tilde{g}_{j}(x)-\tilde{g}_{j-1}(x) & =\int_{t=0}^{1} d \tilde{g}_{j-1}\left(g_{j t}(x)\right)\left[g_{j(2)}\right], \\
\left\|\tilde{g}_{j}-\tilde{g}_{j-1}\right\|_{\rho_{j+1}, 0} & \leq C_{1}\left\|g_{j(2)}\right\|_{\rho_{j+1}, 0} .
\end{aligned}
$$


It follows that $\tilde{g}_{j}$ will converge uniformly on $D_{\infty}$ to a Lipschitz continuous mapping $\tilde{g}_{\infty}$. We further estimate $\tilde{g}_{j}=\tilde{g}_{j-1} \circ g_{j}$ on $D_{\infty}$, using the chain-rule estimate (5.5). Combining some constants gives

$$
\left\|\tilde{g}_{j}\right\|_{k} \leq K_{j}(k)\left(\left\|\tilde{g}_{j-1}\right\|_{k}+C_{1}\left\|g_{j(2)}\right\|_{k}\right)
$$

We use this with $k=2$ to estimate the 1-norm of (14.5). We use the product-rule estimate (5.4) and the chain-rule estimate (5.5) for $\tilde{g}_{j}=\tilde{g}_{j-1} \circ g_{j}$, as in the proof of lemma (5.2). On $D_{\infty}$ we get

$$
\begin{aligned}
\left\|\tilde{g}_{j}-\tilde{g}_{j-1}\right\|_{1} & \leq K_{j}(1)\left(\left\|g_{j(2)}\right\|_{0} \max _{t}\left\|d \tilde{g}_{j-1} \circ g_{j t}\right\|_{1}+C_{1}\left\|g_{j(2)}\right\|_{1}\right), \\
\left\|d \tilde{g}_{j-1} \circ g_{j t}\right\|_{1} & \leq K_{j}(1)\left(\left\|\tilde{g}_{j-1}\right\|_{2}+C_{1}\left\|g_{j(2)}\right\|_{1}\right) \\
\left\|\tilde{g}_{j}\right\|_{2} & \leq K_{j}(2)\left(\left\|\tilde{g}_{j-1}\right\|_{2}+C_{1}\left\|g_{j(2)}\right\|_{2}\right)
\end{aligned}
$$

Using (10.10), (10.9), and (10.7) gives

$$
\left\|g_{j(2)}\right\|_{2} \leq K_{j}(2)\left(t_{j}^{s-(1 / 2)} N_{j}(2)+t_{j}^{k+s-2} N_{j}(k+2)\right) .
$$

Since $k \geq 1$ and $s=2$ the exponents are positive. If we combine all the above, use lemma 11.1 and the ratio test, we see that $\sum\left\|\tilde{g}_{j}-\tilde{g}_{j-1}\right\|_{1}<\infty$. Hence, $\tilde{g}_{j} \rightarrow \tilde{g}_{\infty}$ in $C^{1}\left(D_{\infty}\right)$, and we must show that it has a $C^{1}$ inverse.

From $\tilde{f}_{j}=f_{j} \circ \tilde{f}_{j-1}$, we get as above,

$$
\left|d \tilde{f}_{j}\right|_{\tilde{U}_{j}} \leq \prod_{i=0}^{j}\left(1+\left|d f_{i(2)}\right|_{\rho_{i}}\right) \leq C_{1},
$$

and so $C_{1}^{-1} \leq\left|d \tilde{g}_{j}\right|_{\rho_{j+1}} \leq C_{1}$, for all $j$. Thus $\tilde{g}_{\infty}$ is a $C^{1}$-diffeomorphism of $D_{\infty}$ onto a neighborhood $\tilde{g}_{\infty}\left(D_{\infty}\right)$ of 0 .

We continue the estimation of $\tilde{f}_{j}$ on a convex subdomain $\tilde{U}_{\infty}$ of $\tilde{g}_{\infty}\left(D_{\infty}\right)$ containing 0 . In the next two estimates we use the chain-rule estimate (5.5) and the triangle inequality. In the third we have gone back to (5.8) in order to utilize (8.4) and (8.5).

$$
\begin{aligned}
\tilde{f}_{j}-\tilde{f}_{j-1} & =f_{j(2)} \circ \tilde{f}_{j-1} \\
\left\|\tilde{f}_{j}-\tilde{f}_{j-1}\right\|_{\tilde{U}_{\infty, a}} & \leq K_{j}(a)\left(\left\|f_{j(2)}\right\|_{\tilde{\rho}_{j}(2), a}+\left\|f_{j(2)}\right\|_{\tilde{\rho}_{j}(2), 1}\left\|\tilde{f}_{j-1}\right\|_{\tilde{U}_{\infty}, a}\right), \\
\left\|\tilde{f}_{j}\right\|_{\tilde{U}_{\infty}, a} & \leq K_{j}(a)\left(\left\|f_{j(2)}\right\|_{\tilde{\rho}_{j}(2), a}+2\left\|\tilde{f}_{j-1}\right\|_{\tilde{U}_{\infty}, a}\right) \\
\left\|f_{j(2)}\right\|_{\tilde{\rho}_{j}(2), a} & \leq K_{j}(a)\left(\left\|F_{j}\right\|_{\tilde{\rho}(2), a}+N_{j}(3) \delta_{j}(1) N_{j}(a)\right)
\end{aligned}
$$

In the case $m \in \mathbf{Z}$, we take $k<a<m+1 / 2, a=\lambda k+(1-\lambda)(m+1 / 2)$, $0<\lambda<1$, and we want to show $\sum\left\|\tilde{f}_{j}-\tilde{f}_{j-1}\right\|_{\tilde{U}_{\infty}, a}<\infty$. Combining (14.12) 
and (14.14), using (5.2), and (10.9) with $a=b=1$, and (10.7), we are reduced to showing the following.

$$
\begin{gathered}
\sum K_{j}(a)\left\|F_{j}\right\|_{\tilde{\rho}_{j}(2), a} \leq \sum K_{j}(a)\left(\left\|F_{j}\right\|_{\tilde{\rho}_{j}(2), k}\right)^{\lambda}\left(\left\|F_{j}\right\|_{\tilde{\rho}_{j}(2), m+1 / 2}\right)^{1-\lambda}<\infty, \\
\sum K_{j}(a) N_{j}(3) \delta_{j}(1) N_{j}(m+1 / 2)<\infty \\
\sum K_{j}(a)\left\|f_{j(2)}\right\|_{\tilde{\rho}_{j}(2), 1}\left\|\tilde{f}_{j-1}\right\|_{\tilde{U}_{\infty}, m+1 / 2} \leq \\
\sum K_{j}(a) N_{j}(3) t_{j}^{s-(1 / 2)}\left(1+\left\|\tilde{f}_{j-1}\right\|_{\tilde{U}_{\infty}, m+1 / 2}\right)<\infty .
\end{gathered}
$$

The finiteness of the first two sums follows as in the last two sections. In fact (8.5) with $a=m+(1 / 2)$ and $l=m$, and (10.7) give

$$
\left\|F_{j}\right\|_{\tilde{\rho}_{j}(2), m+(1 / 2)} \leq K_{j}(m+(1 / 2))\left(N_{j}(\beta) \delta_{j}(m)+N_{j}(m+\beta) t_{j}^{s}\right) .
$$

Thus $\left\|F_{j}\right\|_{\tilde{\rho}_{j}(2), m+(1 / 2)}$ and $N_{j}(m+1 / 2)$ are bounded by $B_{j}$, which has the growth (13.18). We also see that $\left\|F_{j}\right\|_{\tilde{\rho}_{j}(2), k}$ and $\delta_{j}(1)$ are bounded by positive powers of $t_{j}$ times slowly growing factors.

For the third we must check the growth of the last factor. Using (14.15) with $a=m+(1 / 2)$, we get

$$
\begin{aligned}
1+\left\|\tilde{f}_{j}\right\|_{\tilde{U}_{\infty}, m+1 / 2} & \leq K_{j}(m+(1 / 2))\left(1+\left\|\tilde{f}_{j-1}\right\|_{\tilde{U}_{\infty}, m+1 / 2}+W_{j-1}\right) \\
W_{j-1} & \equiv\left\|F_{j}\right\|_{\tilde{\rho}_{j}(2), m+1 / 2}+N_{j}(3) t_{j}^{s} N_{j}(m+(1 / 2)) \\
W_{j} & \leq K_{j}(m+(1 / 2)) B_{j} .
\end{aligned}
$$

The last inequality follows from (8.5) with $a=m+(1 / 2), l=m$ and (13.9). We apply the ratio test to (14.19),

$$
\begin{aligned}
\frac{K_{j+1}(a) N_{j+1}(3) t_{j+1}^{s}\left(1+\left\|\tilde{f}_{j}\right\|_{m+1 / 2}\right)}{K_{j}(a) N_{j}(3) t_{j}^{s}\left(1+\left\|\tilde{f}_{j-1}\right\|_{m+1 / 2}\right)} \leq & \hat{c}_{a} 3 K_{j}(3) t_{j}^{(\kappa-1) s} . \\
& \cdot\left(1+K_{j}(m+(1 / 2)) B_{j}\right) \rightarrow 0
\end{aligned}
$$

as $j \rightarrow \infty$.

It follows that the two sequences $\tilde{f}_{j}, \tilde{g}_{j}$ both converge in $C^{a}$-norm, for every $a<m+1 / 2$, on neighborhoods of 0 to inverse $C^{a}$-diffeomorphisms $\tilde{f}_{\infty}, \tilde{g}_{\infty}$.

The mapping $\tilde{f}_{\infty}$ provides a CR equivalence between the original structure $X_{\alpha}$ and the embedded structure $X_{\infty}$. Hence $Z_{\infty} \circ \tilde{f}_{\infty}$ is a CR embedding of class $C^{a}$ of our original structure, for every $a<m+1 / 2$ if $m \in \mathbf{Z}$. In case $m=\infty$, the preceding can be applied, to the same fixed sequence, for every sufficiently large integer $m$ (with perhaps different constants for each $m<\infty$ ). This finishes the proof of theorem 0.1 .

In the case $m \in \mathbf{R}$, the same argument works with $m+(1 / 2)$ replaced by $m$. This gives the proof of proposition 12.1. 


\section{References}

[1] T. Akahori, A new approach to the local embedding theorem of CR-structures for $n \geq 4$, Memoires of AMS, N0. 366, Providence, RI (1987).

[2] L. Boutet de Monvel, Intgration des quations de Cauchy-Riemann induites formelles. Sminaire Goulaouic-Lions-Schwartz 1974-1975; quations aux derives partielles linaires et non linaires, pp. Exp. No. 9, 14 pp. Centre Math., cole Polytech., Paris, 1975.

[3] D. Catlin, Sufficient conditions for the extension of CR structures. J. Geom. Anal. 4 (1994), no. 4, 467-538.

[4] G. Folland and J.J. Kohn, The Neumann problem for the Cauchy-Riemann complex. Annals of Mathematics Studies, No. 75. Princeton University Press, Princeton, N.J.; University of Tokyo Press, Tokyo, 1972.

[5] X. Gong and S. M. Webster, Regularity for the CR vector bundle problem I, Pure and Appl. Math. Quarterly, (to appear).

[6] X. Gong and S. M. Webster, Regularity for the CR vector bundle problem II, pre-print.

[7] G. M. Henkin and Romanov, Exact Holder estimates of the solutions of the $\bar{\delta}$ equation, Izv. Akad. Nauk SSSR Ser. Mat. 35 (1971), 1171-1183; Math USSR Izv. 5 (1971) 1180-1192.

[8] G. M. Henkin, The Lewy equation and analysis on pseudoconvex domains, Russ. Math. Surv. 32 (1977) 59-130.

[9] G. M. Henkin and J. Leiter, Theory of functions on complex manifolds. Monographs in Mathematics, 79. Birkhuser Verlag, Basel, 1984.

[10] L. Hörmander, The boundary problems of physical geodesy. Arch. Rational Mech. Anal. 62 (1976), no. 1, 1-52.

[11] H. Jacobowitz and F. Treves, Nonrealizable CR structures. Invent. Math. 66 (1982), no. 2, 231-249.

[12] N. Kerzman, Hölder and $L^{p}$ estimates for solutions of $\bar{\partial} u=f$ in strongly pseudoconvex domains, CPAM 24 (1971) 301-379.

[13] J. J. Kohn, Boundaries of complex manifolds. 1965 Proc. Conf. Complex Analysis (Minneapolis, 1964) pp. 81-94, Springer, Berlin. 
[14] M. Kuranishi, Strongly pseudoconvex CR structures over small balls. III. An embedding theorem. Ann. of Math. (2) 116 (1982), no. 2, 249-330.

[15] L. Ma and J. Michel, Regularity of local embeddings of strictly pseudoconvex CR structures, J. Reine Angew. Math. 447 (1994) 147-164.

[16] J. K. Moser, A new technique for the construction of solutions of nonlinear differential equations. Proc. Nat. Acad. Sci. U.S.A. 471961 1824-1831.

[17] J. K. Moser, On invariant curves of area preserving mappings of an annulus, Nach. Akad. Wiss. Göttingen, Math. Phys. Kl., Vol. IIa, No. 1, (1962) 1-20.

[18] J. K. Moser, A rapidly convergent iteration method and nonlinear differential equations I, Ann. Scuola Norm. Pisa, 20 (1966) 265-315.

[19] A. Nagel and J-P. Rosay, Non existence of a homotopy formula for $(0,1)$ forms on hypersurfaces in $\mathbf{C}^{3}$, Duke Math. Jour. 58 (1989) 823-827.

[20] L. Nirenberg, Lectures on linear partial differential equations. CBMS Regional Conference No. 17., AMS Providence, R.I., 1973.

[21] R. M. Range, Holomorphic functions and integral representations in several complex variables. Graduate Texts in Mathematics, 108. Springer-Verlag, New York, 1986.

[22] S. M. Webster, A new proof of the Newlander-Nirenberg theorem, Math. Zeit. 201 (1989) 303-316.

[23] S. M. Webster, On the local solution of the tangential Cauchy-Riemann equations, Ann. Inst. H. Poincare, 6 (1989) 167-182.

[24] S. M. Webster, On the proof of Kuranishi's embedding theorem, Ann. Inst. H. Poincare, 6 (1989) 183-207.

Department of Mathematics

University of Wisconsin

gong@math.wisc.edu

Department of Mathematics

University of Chicago

webster@math.uchicago.edu 